\title{
La fabrique d'un saint en Tanzanie post-socialiste
}

Essai d'analyse sur l'Église, l'État et le premier président Julius Nyerere

\section{Marie-Aude Fouéré}

\section{CpenEdition}

Journals

Édition électronique

URL : https://journals.openedition.org/eastafrica/609

DOI : $10.4000 /$ eastafrica.609

ISSN : 2790-1076

Éditeur

IFRA - Institut Français de Recherche en Afrique

Édition imprimée

Date de publication : 1 juin 2008

Pagination : 47-97

ISSN : 2071-7245

\section{Référence électronique}

Marie-Aude Fouéré, «La fabrique d'un saint en Tanzanie post-socialiste », Les Cahiers d'Afrique de l'Est / The East African Review [En ligne], 39 | 2008, mis en ligne le 07 mai 2019, consulté le 13 décembre 2021. URL : http://journals.openedition.org/eastafrica/609 ; DOI : https://doi.org/10.4000/eastafrica. 609

Ce document a été généré automatiquement le 13 décembre 2021.

Les Cahiers d'Afrique de l'Est / The East African Review 


\title{
La fabrique d'un saint en Tanzanie post-socialiste
}

\author{
Essai d'analyse sur l'Église, l'État et le premier président Julius Nyerere
}

\author{
Marie-Aude Fouéré
}

1 En 2005, l'Église catholique de Tanzanie lançait une requête auprès du Vatican pour la canonisation $^{1} \mathrm{du}$ père de l'indépendance et premier président de la République unie de Tanzanie, Julius Kambarage Nyerere (1922-1999). L'instruction d'un procès en canonisation pour un homme politique n'est pas chose courante. Elle paraît d'autant plus étonnante qu'elle concerne un homme qui fut à la tête d'un État régi par des principes laïques. C'est sous l'influence du père de la nation tanzanienne lui-même que, dès l'indépendance du pays puis lors de la Déclaration d'Arusha instaurant le socialisme d'État en 1967, le parti unique d'alors imposa la séparation du politique et du religieux. Toutefois, des liens forts attachèrent toujours ces deux espaces. Les principes des deux religions majoritaires dans le pays, le christianisme et l'islam, furent convoqués pour asseoir le socialisme, et les représentants religieux furent tenus de soutenir la doctrine socialiste en facilitant l'adhésion de la population à la nouvelle voie de développement social et économique. Non dénuées d'ambivalence, les connivences entre le parti unique et l'Église catholique romaine étaient particulièrement marquées. Nyerere, membre actif de l'Église catholique, resta un fervent pratiquant tout au long de sa vie. Il entretint des liens d'amitié forts avec les prêtres de son diocèse et les membres influents du clergé, et requerra une implication forte de l'Église dans l'instauration d'une culture politique socialiste et dans la mise en œuvre des programmes de développement socialistes. L'actuelle ouverture d'une cause en canonisation renvoie clairement aux relations ambiguës entre la sphère politique et le pouvoir catholique depuis l'indépendance. Elle engage à interroger les rapports entre l'Église et l'État dans le contexte politique, social et économique de la Tanzanie postsocialiste, et à rendre compte des imaginaires de la nation remodelés par ce nouveau contexte, en prenant en considération les défis particuliers auxquels ces deux instances de pouvoir sont chacune confrontées. 


\section{Retour sur le processus canonique}

2 Les démarches initiales en vue de la canonisation de l'ancien président Julius Nyerere furent entamées au début des années 2000. La première étape de cette requête, à savoir l'acceptation de l'ouverture d'un procès en canonisation par la Congrégation du Vatican pour la cause des Saints, fut engagée par le diocèse de Musoma - région occidentale de Tanzanie où naquit Nyerere, et où se situe Butiama, le village où il résida pendant ses années d'école primaire ${ }^{2}$, puis qui devint son lieu de résidence après sa retraite et où se trouve aujourd'hui sa sépulture - avec l'appui de l'Église de Tanzanie. À cette époque, cette initiative n'avait pas été rendue publique. Les Tanzaniens n'en apprirent l'existence que six ans plus tard, le 13 janvier 2006. Ce jour-là, feu l'évêque Justin Samba ${ }^{3}$ du diocèse de Musoma annonça en effet que le titre de «serviteur de Dieu » avait été officiellement octroyé à Nyerere par le Vatican en mai 2005. Cela signifiait qu'un procès en canonisation était ouvert. Au cours de cette annonce publique, l'évêque diocésain requerra que toute personne susceptible d'apporter des pièces au dossier de canonisation de Nyerere se présente au diocèse. Prières, contributions financières et témoignages sur la vie pieuse de Nyerere, ou sur des présumés miracles survenus suite à son intercession, au cours de son vivant ou après sa mort, furent sollicités. Une semaine plus tard, le 21 janvier 2006, la messe spéciale qui se tint à Butiama rassembla les évêques catholiques du pays et des dignitaires religieux étrangers impliqués dans le procès en canonisation. La date était symbolique : c'était celle du mariage de Nyerere et de Maria Waningu Majige, célébré au début des années $1950^{4}$. Parmi les dignitaires religieux présents se trouvaient le père Wojciech Koscielniak, postulateur ${ }^{5} \mathrm{du}$ procès en canonisation, le père supérieur Edward Hayes de la congrégation de Maryknoll ${ }^{6}$ (paroisse de Kowak) et vice-postulateur; le père John Civille de l'Archidiocèse de Cincinnati aux USA et co-auteur d'un ouvrage biographique ${ }^{7}$ sur Nyerere, le vicaire général du diocèse, le père Laurenti Magesa, et le père Philbert Rwehumbiza, tous trois les censeurs théologiques ${ }^{8}$ du procès. Célébrée par le cardinal de Dar es Salaam, Mgr Polycap Pengo', cette messe inaugurait officiellement le début du procès en canonisation, la Sessio Prima. Le cardinal y rappela que l'Église de Tanzanie avait présenté la cause de Nyerere auprès du Vatican en se fondant sur deux critères principaux : le respect au quotidien, par Nyerere, des principes de vie chrétiens, et l'influence de sa foi sur sa carrière politique ${ }^{10}$. Le rappel de ces deux critères, valables dans toute introduction d'une cause en canonisation, n'était pas fortuit: il soulignait qu'on entrait, à ce jour, dans un véritable "procès ", au sens légal du terme, qui nécessitait que des preuves et des témoignages soient accumulés.

En effet, le statut de saint n'est attribué qu'au terme d'une procédure rigoureuse, actuellement régie par le Divinus perfectionnis magister du 25 juin 1983 ${ }^{11}$. Après l'attribution du statut de serviteur de Dieu, le procès en canonisation s'ouvre. Il conduit à la vénération - un individu est "vénérable " lorsque sa vie est jugée héroïque. Les investigations en vénération doivent témoigner que le serviteur de Dieu a mené une vie exemplaire, caractérisée par la force de la foi et la pratique au quotidien des vertus chrétiennes. Il peut alors être l'objet d'un culte local - en général, au niveau du diocèse. L'étape suivante, la béatification, qui fait d'un homme un «bienheureux », nécessite la reconnaissance d'un miracle attribué au candidat à la sainteté, qui doit se produire après sa vénération. Sa vie peut alors être donnée en exemple aux chrétiens, et devient l'objet d'un culte public plus large - régional ou national. La dernière étape, celle de la 
canonisation proprement dite, par laquelle un candidat est inscrit sur la liste officielle des saints (le canon), nécessite la reconnaissance d'un second miracle. Le saint est alors l'objet d'un culte universel. Le postulateur de la cause, nommé par l'évêque du diocèse ayant introduit la cause en canonisation auprès du Vatican, secondé par le vicepostulateur, est en charge de promouvoir et de suivre les différents épisodes de la cause. Au cours de la première étape pour la vénération, il est aussi appuyé par deux censeurs théologiques, chargés d'examiner les écrits laissés par le candidat en vénération et montrer comment ils éclairent sa personnalité spirituelle. Un tribunal composé des délégués épiscopaux, comprenant le juge, le promoteur en justice (ou avocat du diable) et le notaire, est en charge de mener l'investigation en recueillant les témoignages sur les vertus chrétiennes du candidat suivant des procédures d'enquête rigoureuses. Les sessions se passent en huis clos. Seul le postulateur peut y être accepté, sans toutefois pouvoir officiellement intervenir. Des commissions d'experts de différentes disciplines sont généralement requises pour apporter des éclairages sur le contexte historique de la vie du candidat. Les actes de l'enquête, qui exposent les preuves de la vie héroïque à partir des déclarations des témoins oraux ou visuels et de la documentation historique recueillie lors de l'investigation, sont portés au Vatican. Le postulateur, secondé par des clercs du Vatican, prépare alors le document final - appelé la Positio super vita et virtutibus ${ }^{12}$ - qui est déposé pour être étudié auprès de la Congrégation pour la cause de Saints. Le verdict en vénération est laissé au vote des cardinaux et des évêques de la Congrégation, avant d'être, au final, porté devant le Pape lui-même pour approbation. Les enquêtes en béatification et en canonisation ne sont menées que si des miracles sont reportés. La canonisation proprement dite se décrète lors d'un consistoire avec les évêques de Rome, et sur décision finale du Pape. $\mathrm{Au}$ regard de cette longue procédure, rien ne permet aujourd'hui d'affirmer que la canonisation de Nyerere est gagnée d'avance, malgré certains propos qui abondent dans ce sens et laissent croire à un débouché certain ${ }^{13}$, bien que long. Certes, la première étape fut passée avec succès. Les écrits de Nyerere, et d'autres documents pertinents, furent examinés, pour aboutir à la conclusion qu'il avait mené une vie héroïque; et des témoins témoignèrent de certains faits indiquant une pratique quotidienne des vertus chrétiennes. Mais le titre de serviteur de Dieu, aisément décerné de part le monde, n'augure en rien d'une suite favorable. La tâche est ardue, et nécessite donc, comme le requerra le cardinal Polycap Pengo dans son discours inaugural, la contribution de tous les citoyens tanzaniens à la cause en sainteté. Le pouvoir divin est même convoqué, l'Église tanzanienne ayant introduit une prière demandant à Dieu de révéler la sainteté de Nyerere ${ }^{14}$.

\section{L'invention contemporaine d'un saint}

4 Nyerere mourut le 14 octobre 1999. Atteint de leucémie chronique depuis plusieurs années, il avait été admis à l'hôpital londonien de Saint Thomas au début du mois d'octobre lorsque son état général s'aggrava. Sa dépouille fut rapatriée en Tanzanie pour être enterrée dans le village de Butiama, dans sa région natale, le 23 octobre 1999. Les funérailles religieuses dont il bénéficia auraient pu mettre la puce à l'oreille d'un observateur attentif que le clergé de Tanzanie n'allait sans doute pas se contenter de cet adieu. Ainsi, la messe de requiem qui eut lieu à la cathédrale Saint Joseph de Dar es Salaam le 20 octobre 1999, six jours après le décès de Nyerere, montra avec évidence que l'Église de Tanzanie faisait grand cas de cet homme politique. Mgr Polycarp Pengo 
ordonna que les vêtements de messe et la nappe de l'autel, de couleur pourpre dans les cérémonies de funérailles, soient blancs - la couleur associée à la résurrection du Christ. Par ailleurs, le chant liturgique de 1'Alléluia de Friedrich Handel, qui accompagne habituellement la saison pascale, fut entonné par la chorale. Ces écarts par rapport aux conventions funéraires traditionnelles signalaient manifestement l'importance, aux yeux de l'Église, de témoigner d'égards tout particuliers à l'ancien président Nyerere. Sachant que selon l'article 9a des Normes pour la cause des saints (Novce leges pro causis sanctorum) promulguées le 7 février 1983, un diocèse ne peut introduire une cause en canonisation qu'après cinq ans révolus après le décès d'un candidat potentiel (ce qui mène, dans le cas de Nyerere, à 2004), il est évident que l'Église de Tanzanie n'a guère perdu de temps pour tenter d'obtenir que cet homme politique de stature internationale, symbole de l'indépendance idéologique et économique de l'Afrique, entre dans la sphère du divin.

5 Depuis l'annonce officielle de l'introduction de la cause en canonisation de Nyerere en 2006, les médias sont restés singulièrement silencieux sur les diverses démarches entreprises par le clergé tanzanien et sur les enquêtes menées par le postulateur et ses collaborateurs. Les preuves et les témoignages recueillis jusqu'à présent pour étayer le dossier n'ont pas été dévoilés au grand public, et il est certain qu'avant la soumission de la Positio aux cardinaux du Vatican, une aura de secret continuera d'entourer les procédures en cours et les résultats atteints. En effet, les investigations pour la canonisation restent secrètes aussi longtemps que la reconnaissance des vertus héroïques du candidat en canonisation n'a pas été effectuée par la congrégation des cardinaux pour la cause des saints. Ce n'est qu'au cours de la dernière commémoration du décès de Nyerere, le 14 octobre de cette année, que, pour la première fois, parut dans un quotidien en anglais un article rédigé par un membre du clergé de Tanzanie, l'évêque auxiliaire de l'archidiocèse de Dar es Salaam, Methodius Kilaini ${ }^{15}$. Dans cet article, M. Kilaini expose les étapes habituelles de tout procès en canonisation, avant de préciser les raisons ayant conduit l'Église catholique de Tanzanie à introduire un procès en canonisation en faveur de Nyerere, et d'exposer les arguments pouvant étayer ce procès - sur lesquels nous revenons dans le paragraphe suivant à travers un compte rendu des articles de presse. S'il n'évite pas la délicate question de savoir si la participation active à la vie politique ne fait pas de facto obstacle à toute possibilité d'actions saintes, l'auteur évite habillement de s'enferrer dans un inventaire du " pour » et du « contre » en rappelant que Nyerere «n'a pas tué ses ennemies politiques (...) comme tant de ses contemporains l'ont fait " ${ }^{16}$, et que les actes politiques de celui-ci doivent être jugés à l'aune du contexte historique dans lequel ils se sont produits. L'article ne dévoile aucune information nouvelle, et se clôt par un appel à témoignages ${ }^{17}$.

Par ailleurs, sans toutefois aborder de front les résultats en cours du procès en canonisation ni prendre position quand à la pertinence de la requête en saintetée ${ }^{18}$, la presse écrite proche des organes du pouvoir, tels les quotidiens Daily News et The Guardian en anglais, ou Nipashe ${ }^{19}$ en swahili, sert nettement la cause en cours. Les exercices de relecture de la vie de Nyerere y fleurissent, éclairant d'une lumière spirituelle les événements marquants de son parcours personnel et politique. Des sites Internet créés par des croyants diffusent eux aussi des biographies élogieuses de Nyerere $^{20}$. Une multitude de ces relectures interprétatives débute par son éducation dans des écoles où Nyerere reçut les premiers éléments de sa formation religieuse. En tant que fils d'un chef du groupe ethnique des Wazanaki, il intégra le système éducatif à 
l'âge de 12 ans, d'abord dans le primaire à Butiama, chez les pères de la société Maryknoll, puis dans le secondaire à Tabora. Il ne se fit baptiser qu'en $1942^{21}$, à l'âge de 20 ans, par des pères Maryknoll dans le diocèse de Musoma. Ce baptême tardif s'expliquerait par le fait que les pères qui suivaient la formation religieuse de Nyerere avaient supposé qu'il succéderait à son père, Nyerere Burito, chef polygame et non chrétien. C'est à son baptême qu'il adopta le prénom de Julius. En 1943, il entra à l'université de Makerere en Ouganda. Il y fonda une société appelée Catholic Action (l'Action Catholique), qui organisait des activités religieuses comme l'animation d'une chorale, des retraites annuelles et des pèlerinages en l'honneur des martyrs chrétiens d'Ouganda ${ }^{22}$. On apprend souvent qu'au cours de ces années d'études à Makerere, Nyerere avait sérieusement envisagé de devenir prêtre ${ }^{23}$. Selon le site de la société des Maryknoll de Tanzanie ${ }^{24}$, ce serait un ami prêtre, le père blanc irlandais Richard Walsh, qui l'en aurait dissuadé pour lui conseiller de s'engager en politique ${ }^{25}$. Le site précise aussi que Nyerere aurait délibérément choisi, une fois obtenu son diplôme en éducation (1945), d'enseigner dans des écoles catholiques, tout d'abord au lycée Saint Mary à Tabora, puis au lycée Saint Francis au sud de Dar es Salaam, qui était alors une institution missionnaire. Après quatre années d'enseignement, il fut sélectionné pour entrer à l'université d'Edinburgh. La presse survole généralement cette période, pourtant fondamentale dans l'engagement politique de Nyerere, mais aussi intense sur le plan spirituel. Le biographe John Hatch indique en effet qu'au cours de ses années passées en Écosse, Nyerere se recueillait souvent dans la solitude d'une église, et écrivait régulièrement au père Walsh à Tabora - sans que l'on sache aujourd'hui la teneur de leurs correspondances. Après l'engagement de Nyerere en politique, au début des années 1950, l'homme s'efface derrière le politicien. Le secret qui entoure sa vie privée et la prudence verbale qui le caractérise rendent difficile toute information précise sur sa vie spirituelle intérieure. La presse tanzanienne acquise à la cause de Nyerere ne peut se mettre sous la dent que des anecdotes relatives à sa pratique religieuse, peu nombreuses. Elle reprend à son compte ce que dit Hatch, dans sa biographie de Nyerere, à savoir qu'il était un catholique dévot: "he has never been merely a nominal member of the church. He has always practised his religion and participated in its observances ${ }^{26}$. Il semble ainsi que Nyerere observait scrupuleusement les périodes de jeûnes imposées par l'église. Un article paru dans The Guardian à l'occasion de la commémoration du décès de Nyerere en 2007 rapporte qu'à une soirée organisée à Butiama en l'honneur de sa retraite, nouvellement prise, Nyerere ne se serait servi que de riz blanc et d'eau parce qu'on était en période de carême. Il se rendait aussi à la messe quotidiennement. On lui attribue à ce sujet les propos suivants, tenus en privé au cardinal Laurian Rugambwa ${ }^{27}$ et que les journalistes citent incessamment : «Without a daily mass, it would have been impossible for me to do my work $»^{28}$. De nombreux articles indiquent qu'il rencontra le pape Jean-Paul II - celui-ci fit une visite en Tanzanie au début du mois de septembre 1990. À cet égard, une photographie de la rencontre entre Nyerere et le Pape est souvent reproduite dans la presse. Certains écrits indiquent que, dans les derniers temps de sa vie, ses amis l'avaient surnommé "l'évangéliste africain », parce qu'il avait traduit des évangiles du Nouveau Testament en swahili ${ }^{29}$. Des ouvrages récents s'ajoutent à la liste des articles de presse voués à la gloire de Nyerere $^{30}$.

7 Dans ces relectures biographiques, le ressort classique qui préside à «l'illusion biographique $»^{31}$, selon laquelle tout individu porterait en lui la nécessité de son futur, est activé. La cohérence rétrospective de la vie de Nyerere est créée par ces interprètes 
biographes, qui listent en vrac les principes cachés qui auraient déterminé, au sens fort de ce terme, son parcours individuel. Ces principes seraient non seulement moraux, mais surtout divins. En effet, les exercices de reconstruction biographique décrits laissent dans l'ombre Nyerere le politicien, pour mieux mettre l'accent sur Nyerere le croyant. La politique n'apparaît que sous la forme des principes humanistes (justice, égalité, liberté) qui, pendant la période nyérériste, étaient convoqués pour asseoir l'idéologie socialiste ${ }^{32}$, et les associent aux grandes valeurs chrétiennes. Bien loin de l'explication sociologique selon laquelle tout individu est le produit de son temps, ces réécritures biographiques de la vie de Nyerere mettent l'accent sur la transcendance religieuse pour expliquer sa personnalité, ses actes, et ses décisions politiques. Dans cette perspective, ce ne serait pas l'esprit panafricaniste de l'époque, la période de l'indépendance dans l'ensemble du monde colonisé, le contexte de la guerre froide et les efforts de non-alignement qui constitueraient des facteurs à prendre en compte pour comprendre son parcours personnel et politique. Au contraire, ce parcours original serait l'unique effet d'une foi incommensurable, fruit de l'enseignement religieux reçu dans son enfance et auquel la main de Dieu ne serait pas étrangère. Comme dans tous les cas d'invention de héros fondateurs, la figure de Nyerere est produite par le biais d'une décontextualisation historique et d'une visée eschatologique. Ainsi seulement peut-elle accéder à l'universel.

\subsection{L'invention contemporaine d'un mythe national}

8 L'annonce de l'introduction d'un procès en canonisation en faveur de Nyerere, aussi surprenante puisse-t-elle paraître à première vue, ne s'est pas produite en terrain vierge. En effet, depuis le décès du premier président tanzanien en octobre 1999, on assiste à une surenchère de louanges commémoratives qui, au cours des trois dernières années, a atteint un seuil sans précédent dans les médias les plus en vue. Chaque nouveau mois d'octobre, la commémoration de sa disparition est l'occasion, pour la presse proche du pouvoir, de déployer tout un arsenal médiatique voué à sa gloire. La réactivation du souvenir du "Baba wa Taifa " (le " père de la nation ", en swahili), qui se fait sur le mode de l'hommage respectueux et de l'hagiographie, est voué à opérer une mythification de l'homme ${ }^{33}$. Pour ce faire, différents procédés sont mis en œuvre par les médias. Les nombreux articles de journaux à teneur biographique déclinent ses qualités personnelles sur le mode du panégyrique: simplicité, modestie, sobriété, honnêteté, détermination, charisme. Les événements marquants de sa vie, ses actes et les propos tenus en certaines occasions publiques sont interprétés comme des révélateurs des traits fondamentaux, et toujours présentés comme exceptionnels, de sa personnalité. Il est constamment souligné que l'appellation de «mwalimu» (le maître d'école), utilisée pour désigner l'ancien président, ne serait pas seulement due à sa profession d'enseignant. Elle serait le produit de l'humilité de Nyerere, qui aurait luimême choisi ce terme contre les prétentieuses appellations, prisées par de nombreux dirigeants africains, de "sa sainteté » ou d'«empereur». De plus, les articles qui traitent plus spécifiquement du parcours politique de Nyerere s'attachent longuement à présenter les valeurs dont il s'était fait le défenseur tout au long de sa vie de politicien. Les termes de liberté, de justice, d'unité et d'égalité, largement employés par l'ancien président dans ses écrits politiques ${ }^{34}$, reviennent sans cesse. L'histoire politique du pays est présentée comme une succession de tentatives d'application des principes moraux chers à Nyerere, et non comme le résultat du pragmatisme stratégique habituel 
dans le monde politique, peu soucieux de morale humaniste ${ }^{35}$. La couverture médiatique des commémorations du décès de Nyerere témoigne à l'évidence d'un travail d'invention mémorielle. Enrayant les mécanismes de l'oubli, déjouant les autres modes de souvenir possibles, elle cherche à produire une mémoire nationale univoque du premier président de Tanzanie, héros national ou «titan ${ }^{36}$ incomparable qui fonctionnerait comme un nouveau mythe fondateur de la nation tanzanienne ${ }^{37}$.

Il est remarquable que, non content de jouer avec les ressorts classiques de la construction nationale, par l'invention de figures et de faits héroïques qui gomment la pluralité et la contraction, le processus de mythification auquel le personnage de Nyerere est soumis dans les médias joue avec le répertoire des émotions et ressasse des métaphores sacralisantes pour opérer un basculement du profane au sacré. Un pathétique facile est activé, renouant avec des émotions passées pour les raviver dans le présent. Les descriptions des scènes de vibrante ferveur populaire, qui ont eu lieu à l'occasion du rapatriement de sa dépouille, usent d'hyperboles sur la tristesse et la douleur pour mieux faire vibrer la corde émotionnelle. En ce sens, la référence contemporaine à Nyerere participe d'une politique de l'émotion, où émouvoir (et être ému) est le ressort de l'entretien de l'identité nationale. En outre, une étude de la presse atteste des tentatives de passage du père de la nation du domaine profane, celui de la politique, au domaine du sacré - cela, dès avant la récente annonce officielle (2006) du début du procès en canonisation. Les faits biographiques du chef d'État sont présentés, dans une perspective téléologique évidente, comme les signes d'une prédestination. Son enfance, son adolescence, sa vie d'adulte sont décryptées pour y lire les signes précurseurs d'une prédisposition à gouverner. De plus, de nombreux écrits ont recours à la métaphore de la lumière pour dépeindre Nyerere et son œuvre. Parce que la lumière évoque la connaissance véridique et juste, elle confère à Nyerere une autorité indiscutable dans son travail de guide national. Mais, parce que la métaphore de la lumière renvoie aussi à la vérité révélée, elle opère un glissement du mondain au divin. Le père de la nation acquiert ainsi le statut de sauveur d'un peuple opprimé, comme le furent les prophètes ${ }^{38}$, et le Christ. Dans cette logique, il n'est pas surprenant de voir les médias dépeindre l'implication politique de Nyerere sur le mode de l'abnégation de soi. C'est pour sauver la nation tanzanienne du tribalisme, du racisme, de la pauvreté et de la dépendance politique, économique et culturelle, que Nyerere aurait fait don de sa personne au peuple tanzanien. Ces images métaphoriques du sacrifice puisent dans la liturgie catholique pour asseoir l'analogie entre Nyerere et le Messie chrétien. Les valeurs de justice, d'égalité, et de liberté défendues par Nyerere sont rattachées aux grands principes moraux chrétiens. Les exercices de relecture spirituelle de la vie de Nyerere précédemment décrits, qui avaient précédé l'annonce du procès en canonisation et ont pris une réelle ampleur depuis lors (comme décrit précédemment), s'inscrivent dans cette configuration rhétorique déployée par les médias proches du pouvoir.

\subsection{Aux sources du mythe Nyerere : la période socialiste}

Les pratiques contemporaines de réinvention d'une figure immaculée, impulsées par le pouvoir, se greffent sur le travail de production d'un imaginaire national entamé pendant la période socialiste. Sans présenter en détail les travaux de science politique bien connus sur le "socialisme à l'africaine" (ujamaa en swahili) qu'inaugura la Déclaration d'Arusha en 1967, trois ans après la formation de la République unie de 
Tanzanie ${ }^{39}$, et qui guida le pays pendant près de vingt ans, il convient de rappeler ici les différents ressorts qui furent activés pour faciliter l'adhésion de la population aux principes socialistes et ainsi contribuer à "l'invention d'une culture politique »40 nationale. Il s'agit pour cela de puiser dans les principes religieux et humanistes à portée universelle ${ }^{41}$, d'avoir recours au pouvoir unificateur du partage d'une même langue sur tout le territoire, ceci par l'adoption du swahili comme langue nationale et langue d'enseignement ${ }^{42}$, de revendiquer des racines africaines, réelles ou imaginaires, de la tradition, de l'entraide coutumière et de la solidarité intrafamiliale, qui avaient été fragilisées par le colonialisme ${ }^{43}$, et enfin de s'appuyer sur les aspirations au changement que l'indépendance avait exacerbées par une insistance répétée sur les valeurs de liberté, d'égalité et de justice. Les arts et la culture furent mis à contribution pour diffuser le message socialiste ${ }^{44}$. La promotion des spectacles de danses et de tambours (ngoma) fut justifiée par un retour aux sources culturelles précoloniales, et favorisa la popularisation des valeurs de la nouvelle nation. Ces valeurs s'incarnèrent par ailleurs dans des mesures politiques, sociales et économiques fondées sur la participation de tous au développement du pays. L'exploitation rationnelle des ressources nationales, par la propriété commune des moyens de production, devait assurer un bien-être généralisé et l'autosuffisance du pays - kujitegemea en swahili, traduit par l'expression « compter sur ses propres forces ». L'appropriation privée des moyens de production était présentée comme la pire figure de l'exploitation de l'homme par l'homme. Les mesures économiques et sociales qui furent mises en œuvre reflétaient ces fondements moraux de ce socialisme à l'africaine. Dans le secteur rural, la politique dite de "villagisation » fut adoptée pour placer les moyens de production dans les mains des paysans et assurer l'amélioration des services sociaux. Elle consistait concrètement dans le regroupement des populations au sein de villages communautaires de production agricole, les villages ujamaa. Dans les secteurs industriels, commerciaux et bancaires, un vaste programme de nationalisation fut opéré à partir de 1967. La nationalisation des entreprises privées et des sociétés coopératives de commercialisation précéda de peu celle des biens immobiliers privés puis des grandes plantations commerciales. Enfin, sur le plan social, le gouvernement et le parti s'attachèrent à réformer le système éducatif pour l'adapter aux exigences de la vie villageoise et rurale, et faciliter l'enseignement pour les adultes. L'accès aux services sociaux de base (eau potable, écoles, dispensaires), gratuits, fut généralisé. L'ujamaa se présenta donc comme un modèle de socialisme spécifique à l'Afrique, troisième voie entre les deux modèles économiques et politiques de l'époque, le libéralisme occidental et le communisme de type soviétique.

11 Le chef d'orchestre de ce plan d'ensemble fut le parti unique, qui pendant près de 20 ans de pouvoir, d'abord sous l'appellation de la TANU (Tanganyika African National Union, ou Union nationale africaine du Tanganyika) puis, à partir de 1977, de CCM (Chama Cha Mapinduzi, ou Parti de la révolution), parvint à mettre en place des moyens de contrôle qui s'étendaient à l'ensemble de la vie politique du pays. À la fois la prégnance de l'idéologie ujamaa et le recours à un contrôle politique et social fort donnèrent forme à une culture politique originale, qui fut louée dans les médias nationaux et internationaux $\mathrm{x}^{45}$. Au sein du parti unique, le président Nyerere fut le rouage essentiel du système ujamaa. Il le fut tout d'abord en tant qu'idéologue capable de formuler les principes socialistes avec clarté et conviction, aussi bien sous forme orale que sous forme écrite ${ }^{46}$. Ses discours publics et ses nombreux écrits politiques - qui témoignent d'une éducation de haut niveau, de sa capacité à articuler habilement des idées 
complexes et des concepts sociaux, économiques et politiques, d'une pensée critique, usant des outils de son temps pour analyser les principes des grandes idéologies d'alors, et encore d'une capacité à opérer un retour réflexif sur ses propres décisions en matière de politique économique et sociale, en relation avec le contexte international ${ }^{47}$ - étaient présentés comme le produit d'un politicien-philosophe à la pensée foncièrement unique et originale. Ses qualités d'orateur et d'expert en swahili furent incessamment louées, alors que les grands auteurs tanzaniens tels Shaaban Robert, Mathias Mnyampala, Ebrahim Hussein ${ }^{48}$ ou encore E. Kezilahabi, certes étudiés dans les écoles du pays, ne bénéficièrent pas d'une telle reconnaissance publique. La publicité faite à ses traductions des grands drames shakespeariens en témoigne ${ }^{49}$. Nyerere fut aussi un rouage essentiel du système ujamaa en tant que détenteur d'un pouvoir fondé sur des liens particuliers qu'il avait développés avec un noyau de fidèles. Ses détracteurs et opposants eurent à souffrir d'être devenus les ennemis du pouvoir, exclus de la sphère politique monopolisée par le parti unique et parfois mis en prison pour rendre impossible leur influence dans la classe politique et sur la population. Maître d'œuvre du socialisme à l'africaine et de sa diffusion idéologique, le président Nyerere fut aussi l'acteur de sa propre héroïsation, moins par vanité personnelle que comme stratégie symbolique pour atteindre les objectifs sociaux et économiques fixés. Pour ce faire, le contrôle des médias fut un outil puissant de la mythification de Nyerere. Ses paroles et ses actes (ses déplacements, ses visites, ses interventions publiques) étaient constamment rediffusés par des médias d'où la critique était exclue. Rappelons qu'avant les années 1990, où les médias se multiplièrent et se diversifièrent, et où une certaine liberté de ton fut rendue possible, la presse et la radio étaient totalement contrôlées par l'État, et la télévision, en Tanzanie continentale tout du moins, interdite. Le Newspaper Ordinance Bill de 1968 donnait le droit au président d'interdire les publications jugées subversives. Les titres de presse appartenaient soit à la société nationale de la Tanganyika Standard Ltd, créée en 1970, tels le Standard, le Daily News et le Sunday News, soit au parti unique, Uhuru (Liberté) et Mzalendo (Le patriote) les quelques autres titres étant rattachés aux syndicats (Mfanyakazi, Le travailleur), aux ministères (Nchi yetu, Notre pays), ou à l'Église catholique (Kiongozi, Le leader/Le dirigeant $)^{50}$. Comme le dit Nyerere lui-même, «The new Standard will be a socialist paper. It will support the socialist ideology of Tanzania as defined in the Arusha Declaration $»^{51}$. Le Newspaper Act de 1976 acheva de museler les medias en imposant que les directeurs des grands titres soient désignés par le Président lui-même, et en imposant qu'ils soient supervisés par un sous-comité rattaché au parti unique et que l'information soit alimentée par une agence rattachée au Ministère de l'Information ${ }^{52}$. Il est vrai que, comme le rappelle Denis-Constant Martin, « les journalistes n'ont jamais vraiment accepté d'être tenus en laisse $»^{53}$. Des tendances gauchistes s'exprimaient à travers les médias, et certains hommes clés du monde de la presse dénoncèrent les pratiques autoritaires $d u$ pouvoir : violation du droit et de la loi, abus de pouvoir, exploitation des travailleurs, corruption et pratiques d'influence. Mais ces manquements, attribués à la bureaucratie, aux entreprises privées ou à certains hommes politiques, ne prenaient pas pour cible directe le président Nyerere. Ce dernier continuait à être présenté comme l'unique artisan de la libération du pays, comme un philosophe original et comme personnalité à part dans le paysage politique africain, par son intégrité et son absence d'ambition personnelle. Son image fut donc, de son temps, l'objet d'une campagne de communication destinée à asseoir sa domination idéologique et son pouvoir politique. Avant de s'interroger sur l'enracinement populaire du grand récit national nyérériste, 
un retour historique sur les rapports entre le pouvoir politique et la religion pendant la période socialiste s'impose.

\subsection{L'État et l'Église pendant la période socialiste}

12 Une des originalités du socialisme à l'africaine développé par Nyerere tient à la place qui est attribuée au religieux dans la société. Rejetant les tendances anti-religieuses et anti-cléricales du socialisme européen, et encore plus la vision marxiste de la religion comme « opium » d'un peuple aveuglé par des faux espoirs de juste redistribution après la mort, Nyerere fit preuve de modération et de pragmatisme politique. Fervent croyant, passé par le système de scolarisation missionnaire, il n'était pas désireux d'apostasier la religion catholique pour troquer l'athéisme contre la foi. Par ailleurs, dans un paysage religieux qui se composait de $40 \%$ de musulmans, $40 \%$ de chrétiens et $20 \%$ de fidèles des religions dites traditionnelles, et où la religiosité était massive, les chances d'une adhésion populaire aux principes antireligieux étaient nulles. Il n'aurait pas été plus tactique de ne reconnaître qu'une seule religion d'État, sauf à vouloir attiser les conflits religieux entre deux communautés d'égale importance. La constitution de 1965, qui instaurait la nouvelle République, décréta donc la séparation de l'Église et de l'État sur le modèle existant dans les démocraties européennes. La laïcité, par laquelle la pratique religieuse restait cantonnée à la sphère privée et la liberté de conscience était reconnue, fut le maître mot de cet État séculier en construction ${ }^{54}$. L'un des premiers slogans politiques du parti unique, la TANU - et ceci dès la période de la lutte pour l'indépendance du pays, alors que le parti n'était encore qu'un mouvement de libération nationale - fut de ne pas mélanger la religion et la politique («Don't mix religion with politics»). Ce slogan s'adressait principalement à la communauté musulmane, qui, au début des années 1960, faisait compétition à l'option socialiste en cherchant à promouvoir l'installation d'un mode de gouvernement musulman. Dans le quotidien du parti unique The Nationalist de 1965, un éditorial intitulé « Religion and Politics » rappelait que : «History has shown how disastrous it is to mix politics and religion. (...) That is why it is imperative that religion must be isolated from the political life of our country ${ }^{55}$. " La séparation du politique et du religieux fut annoncée comme un principe central de la culture politique du pays, en vue de la construction nationale et de la cohésion sociale. En ce sens, il y eut bien une sécularisation de l'espace politique par la séparation des structures religieuses et des institutions politiques, par la mise en place d'un ordre politique qui ne fondait pas sa légitimité sur la volonté divine, mais sur la volonté des hommes, et par l'extension des juridictions de l'État sur les affaires autrefois régulées par la religion ${ }^{56}$.

13 Pourtant, de fait, les références constantes à l'imagerie religieuse dans la vie politique et la mobilisation des mouvements religieux dans le nouvel État en formation ne manquèrent pas de faire apparaître les ambiguïtés du message laïc officiel. Pour que l'élan national qui avait porté la décolonisation puisse être impulsé et pérennisé, par l'invention et la construction de la nation tanzanienne, il convenait d'adopter une idéologie efficace qui puisse remporter l'adhésion de la population. Pour ce faire, les "symboles d'une éthique nationale $»^{57}$ furent sélectionnés dans différents répertoires pour s'ajuster au pluralisme religieux : la tradition africaine, l'humanisme universel, et la morale religieuse. Une véritable religiosité publique prit forme et fut entretenue, qui intégrait des éléments de l'islam et du christianisme ${ }^{58}$. Le calendrier national et les pratiques gouvernementales qui se mirent en place en attestent ${ }^{59}$. En effet, de 
nombreux jours fériés furent mis en place, qui associaient les fêtes religieuses musulmanes, comme l'Idi el fitr, et Idi el haji, ou encore Maulidi pour l'anniversaire du prophète Mahomet, le calendrier chrétien (Vendredi Saint, Pâques, Noël), et les nouvelles fêtes séculières liées à la formation de la nation tanzanienne, telles la célébration de la naissance de la TANU (sabasaba, le 7 juillet), de l'indépendance et de la République ( 9 décembre) ; le jour de la Révolution de Zanzibar ( 12 janvier), la fête de l'Union entre le Tanganyika et Zanzibar (26 avril); et la fête du travail ( $1^{\mathrm{er}}$ mai). Plus remarquable, le recours à des pratiques rhétoriques puisés dans les liturgies cultuelles et l'usage massif du champ sémantique du divin dans les discours publics furent caractéristiques du nouveau symbolisme d'État. Ils furent emblématiques de cette religiosité intégrée à la vie publique civile. Ainsi, l'hymne national tanzanien, joué chaque matin et chaque soir à la radio, et dans toutes les occasions officielles, enjoignait (et enjoint toujours) à Dieu de bénir l'Afrique et la Tanzanie. Une prière œcuménique, intégrant des valeurs morales acceptables pour tous, débutait les sessions parlementaires. Les meetings politiques avec la population s'ouvraient eux aussi par des prières appelant à la protection et à l'aide de Dieu. La mise en valeur des principes moraux directement associés aux grandes religions et les références constantes à un Dieu protecteur de la nouvelle nation agirent à deux niveaux. D'un côté, elles permirent une acceptation aisée de l'ujamaa, en coulant cette vision nationale dans le moule sémantique du religieux, et en lui apportant une caution sacrée. En ceci, le pouvoir politique tanzanien exemplifie cette association étroite entre le pouvoir et le sacré, qui repose sur un partage de même d'attributs, notamment la " prétention à la suprématie et à l'éternité ", et les "pouvoirs surnaturels inhérents au prince comme au prêtre " D'un autre côté, elles contribuèrent à mettre en place un "climat œcuménique " favorable à une bonne entente entre les différentes communautés religieuses ${ }^{61}$.

Par ailleurs, la TANU attendit des représentants des communautés religieuses chrétiennes et musulmanes qu'ils soutiennent l'ujamaa de manière inconditionnelle, aussi bien dans son versant social et économique que dans sa visée idéologique et nationaliste. L'injonction selon laquelle les organisations religieuses devaient «jouer leur rôle» ("Play your part») appelait à leur engagement dans la construction nationale, cela dans les cadres imposés par l'ujamaa. Cette injonction était principalement adressée aux représentants de l'Église catholique. Comme le rappelle Sylvain Urfer, "Nyerere, en catholique sincère, pousse les Églises à s'engager plus en avant dans la tâche du développement, où il estime leur apport irremplaçable $»^{62}$. Ceci s'explique pour deux raisons : les sociétés missionnaires étaient plus engagées dans la conversion spirituelle que dans la promotion du développement économique ou social des populations locales; et elles bénéficiaient de larges soutiens financiers via leurs maisons mères, dont le nouveau gouvernement pouvait tirer avantage ${ }^{63}$. Dans un discours tenu devant les membres de la Société Maryknoll à New York, en 1970, Nyerere développa l'idée que les institutions de l'Église devaient participer activement à libérer les hommes de toutes formes d'oppression. C'est dans ce combat que l'Église trouverait sa raison d'être dans le monde moderne, et qu'elle contribuerait à empêcher que le christianisme ne dégénère en un ensemble de superstitions ${ }^{64}$. Il semble même que Nyerere aurait instamment demandé que les séminaires soient des lieux de formation à la doctrine socialiste, pour que la Tanzanie se dote de "prêtres socialistes $»^{65}$ prompts à propager les principes fondamentaux de l'ujamaa. ${ }^{66}$. Toutefois, malgré ces requêtes incessantes d'une implication active de l'Église, "l'adhésion chrétienne à l'idéologie de l'ujamaa a été à géométrie variable ${ }^{67}$, fonction des interlocuteurs 
et des circonstances. L'Église catholique fut tout d'abord portée à contribuer à l'effort national dans les premières années qui suivirent l'indépendance, où les remèdes recherchés au sous-développement rentraient dans le cadre d'une économie capitaliste. Mais à la déclaration d'Arusha du 5 février 1967, charte des nouveaux principes socialistes, l'Église devint réticente à jouer le rôle qui lui était assigné, tant les directions données à la nouvelle nation tanzanienne menaçaient de porter atteinte à ses biens fonciers et mettaient à mal son autorité et sa puissance financière, fruits de pratiques capitalistes anciennes. Au final, et malgré des oppositions ouvertes parmi les membres étrangers du clergé catholique, les religieux haut placés de l'Église décidèrent pourtant de soutenir officiellement le socialisme à l'africaine. Comme le rapporte D. Westerlung, certains religieux ayant des fonctions politiques au sein de la TANU tentèrent même d'assimiler le christianisme et l'ujamaa, en arguant qu'ils reposaient tous deux sur les mêmes valeurs, et tendaient vers les mêmes objectifs' ${ }^{68}$. Selon lui, on pourrait même parler d'une tentative de "christianisation de l'ujamaa ». Bref, comme le note F. Ludwig6", "in general, the churches followed the government's appeal to "play their part" and laid great emphasis on their duty to help in the process of building up the nation. They promoted unity, stability and harmony; open disagreements with political leaders were avoided». En contrepartie, il semble qu'elles purent obtenir des faveurs du monde politique, et de Nyerere en particulier ${ }^{70}$. Mais le soutien demandé aux dirigeants des grandes instances religieuses allait aussi de pair avec le contrôle de leurs activités. Trois instances représentatives furent créées afin de faciliter ce contrôle: pour les chrétiens, la Tanzanian Episcopal Conference (TEC), organisation catholique, et le Christian Council of Tanzania (CCT), rassemblant les principales Églises protestantes (luthériens, anglicans et moraves); pour les musulmans, le Baraza Ku la Waislamu Tanzania (BAKWATA). Ce contrôle avait pour but d'éviter que les organisations religieuses engendrent de la contestation politique, ou favorisent les divisions religieuses à l'échelle nationale.

Il apparaît clairement que les deux grandes religions musulmane et chrétienne, et surtout l'Église catholique, servirent d'instruments de légitimation de la vérité socialiste délivrée par l'État. C'est pour cette raison que si, selon D. Westerlund, on peut dire que l'État tanzanien s'engagea dans un processus de sécularisation du pouvoir politique, qui eut pour conséquence un déclin de l'influence des religieux dans les modes de gouvernance, il n'en reste pas moins que ce processus ne fut que partiel. La TANU instaura de fait ce qu'il convient d'appeler une "religion civile », c'est-à-dire une forme de religiosité détachée de toute religion particulière, mais ancrée dans le partage de principes moraux de portée universelle, commun aux grandes religions (paix, tolérance, justice, solidarité, etc.) afin de créer un sentiment d'unité sur le plan national. En d'autres termes, l'État socialiste tanzanien usa moins des ressources de la religion comme ensemble de croyances instituées protégées par des détenteurs attitrés, que du religieux ou du sacré comme système de significations pour asseoir son discours nationaliste, et ainsi peser sur les représentations populaires de la nation. En cela, il fut fidèle à cet attribut caractéristique de tout pouvoir politique qui est, comme le rappelle Georges Balandier, "d'entretenir sous forme manifeste ou masquée une véritable religion politique $»^{71}$. 


\subsection{Perte de légitimité politique et concurrence religieuse en Tanzanie post-socialiste}

16 Les liens étroits et privilégiés entre le pouvoir et l'Église, qui caractérisaient la période socialiste malgré la séparation officielle de l'État et de la religion, furent modifiés suite aux changements du paysage économique et politique au milieu des années 1980. À cette époque, le pays tourna le dos aux politiques ujamaa, incapables de créer le développement social et économique promis. Il y eut certes des résultats positifs dans le domaine des services publics, de la santé et de l'enseignement. Mais ils furent en partie masqués par des insuffisances économiques flagrantes, tels un fort exode rural causé par l'insuccès de la villagisation, l'insuffisance des réseaux et des moyens de transport, ou encore l'incompétence des entreprises para-étatiques et leurs dérives vers la corruption. Ces nombreux échecs internes, conjugués aux évolutions du contexte économique international, menèrent à un changement brutal de direction. En 1985, l'année où Nyerere quitta le pouvoir et laissa la place au nouveau président Ali Hassan Mwinyi, le pays opéra un passage radical, bien que préparé depuis le milieu des années 1970 par diverses mesures d'assouplissement des politiques socialistes ${ }^{72}$, d'une économie socialiste planifiée à une économie de libre marché et à la démocratisation de la vie politique. L'adoption d'un système capitaliste requit la mise en œuvre, sous la tutelle de la Banque Mondiale, de programmes de dérégulation économiques fondés sur des mesures d'austérité visant à la réduction des dépenses publiques, au contrôle la masse monétaire et au renforcement des exportations (Economic Recovery Programme, 1986 ; Structural Adjustment Facility, 1987 ; Enhanced Structural Adjustment Facility, 1991). C'est dans ce cadre que s'opérèrent l'ouverture des capitaux des entreprises, la privatisation des industries et entreprises para-étatiques, ainsi que la liberté de création d'entreprises et de banques à titre privé. La mise en place d'un système politique multipartiste (1992), qui mit fin au monopole du CCM dans la vie politique, induisit la reconnaissance de partis politiques concurrents, en phase d'organisation depuis les années 1980. Ces nouveaux partis, comme le Civic United Front (CUF), le Chama cha Demokrasia na Maendeleo (Chadema), l'United Democratic Party (UDP), le National Convention for Construction and Reform-Mageuzi (NCCR-Mageuzi) déployèrent rapidement une activité intense pour concurrencer le CCM Depuis cette époque, s'ils ne ménagent pas leurs critiques envers le C.C.M., ces partis restent faibles et divisés, et n'arrivent pas à dégager de programme politique cohérent. La grande popularité du CCM constitue un monopole de fait, qui s'est traduit par la nette victoire des candidats du CCM, à savoir Benjamin Mkapa en 1995 et 2000, puis Jakaya Mrisho Kikwete en 2005, aux différentes élections présidentielles multipartistes, menées avec une relative transparence. La démocratisation de la vie politique s'accompagna aussi de mesures qui accordèrent l'autonomie des syndicats et des coopératives, et renforcèrent le pouvoir du Parlement face à l'exécutif. Elles accordèrent le droit associatif, le droit de rassemblement et à la liberté de la presse.

17 Ces changements politiques et économiques bouleversèrent les rapports socioéconomiques, modifiant les représentations du pouvoir et, in fine, les valeurs nationales qui avaient soutenu le système politique socialiste. En effet, l'entrée dans une économie capitaliste, qui s'accompagne depuis quelques années d'un taux de croissance fort (7\% par an) et a rendu possible l'émergence d'une classe moyenne dans les grands centres urbains du pays eut en effet pour contrepartie un accroissement de la paupérisation 
des populations les plus vulnérables, touchées par le démantèlement des services sociaux autrefois pourvus gratuitement par l'État socialiste (santé, enseignement primaire). Les bénéfices que les réformes économiques permettent de dégager ne contribuent pas tant au développement homogène du territoire qu'à creuser les inégalités socio-économiques entre urbains et ruraux, et entre le secteur informel et le monde salarié ${ }^{73}$. Par ailleurs, une collusion très forte se développe entre la classe politique et le monde économique et financier, favorisant l'enrichissement d'une petite classe bourgeoise issue des anciennes entreprises para-étatiques et de l'ancien parti unique. La libéralisation de la vie publique et le développement des médias ont donné une grande visibilité aux affaires de grande corruption (ufisadi en swahili). Deux récents scandales, celui de la Banque de Tanzanie et celui de la compagnie Richmond, viennent d'étaler au grand jour le linge sale des organes gouvernementaux et des services publics $^{74}$. Certes, ces deux affaires ont débouché sur une vague de démissions sans précédent de ministres, de membres du cabinet ministériel et de hauts fonctionnaires. Ces opérations de nettoyage de la classe politique ont permis au président Jakaya Mrisho Kikwete de se débarrasser des éléments qui nuisaient à son image de réformateur et d'incorruptible, et ainsi de renforcer sa popularité. Élu sur un programme de lutte contre la corruption, il a ainsi montré qu'il était à la hauteur de ses promesses en se débarrassant des vieux pontes de la politique. Mais la population craint aujourd'hui que ces deux scandales ne forment que la partie émergée de l'iceberg. Au final, la domination idéologique de l'État, qui avait déjà été ébranlée par les échecs économiques de l'ujamaa, est aujourd'hui totalement mise à mal. Le discrédit dont souffrent les élites politiques a atteint un seuil sans précédent, tous les dirigeants étant considérés comme corrompus ${ }^{75}$. Comme l'explique Athumani Liviga " $L a$ fin de l'ujamaa a créé un vide en termes d'idéologie et de stratégie de développement alternatives et (...) ni le parti au pouvoir, ni les partis d'opposition n'ont été capables de mettre en cuuvre, ni même de suggérer aucun autre plan concret pour assurer la réussite économique, sociale, culturelle et politique du pays $»^{76}$.

Parallèlement à la perte de la légitimité politique de l'État tanzanien, les églises traditionnelles issues des missions catholiques et protestantes installées avant et pendant la période coloniale commencèrent à être concurrencées par l'essor et l'influence des nouveaux mouvements religieux en Tanzanie. Il est vrai que, comme le rappelle Friedrich Ludwig pour la Tanzanie, la vague de revivalisme protestant est ancienne ${ }^{77}$. Datant des années 1980, elle est moins un effet de l'ouverture pluraliste que des campagnes d'évangélisation menées par les églises protestantes américaines. Mais depuis la libéralisation politique des années 1990, la visibilité de ces différents groupes et de leurs activités s'est accrue. Le succès du pentecôtisme et son expansion ne sont pas sans lien avec le nouveau contexte économique et politique qui prend forme à la fin de la période ujamaa. Par sa capacité à s'adapter aux cultures et aux situations locales, mais aussi à promouvoir le succès individuel et l'enrichissement financier, elle se moule dans les nouvelles valeurs nationales de compétition, d'individualisme et de succès économique, en même temps qu'elle propose des remèdes aux failles du nouveau système économique : sentiment d'appartenance communautaire lorsque le lien social se délite; promesses de guérison en épidémie de Sida, alors que le coût des soins est devenu prohibitif pour la majorité de la population. En ce sens, ces nouvelles églises créèrent des dynamiques sociales qui, aujourd'hui encore, produisent une «culture modernisante", selon l'expression de Comi Toulabor $^{78}$ dans son travail sur les nouvelles églises du Ghana. On peut compter, dans le cadre du déploiement de ces 
nouvelles églises, l'excommunication du père Felician Nkwera par l'Église catholique et la création du Marian Faith Healing Ministry (MFHM) ${ }^{79}$. Ancien prêtre de la région de Njombe, dans le sud ouest de la Tanzanie, le père Felician Nkwera se fit connaître pour ces activités curatives et exorcistes à la fin des années 1970 et dans les années 1980. Sa popularité croissante, son refus de requérir l'autorisation de la Tanzanian Episcopal Conference pour exorciser ses fidèles, sa remise en cause de la hiérarchie catholique attisa très tôt les craintes des représentants de l'Église. Au sujet de ce dernier point, Nkwera écrivit que : "The church must be destroyed by any means. We are starting at the top. We must destroy her in order to achieve the superpower of mankind "\$0. Quant au cardinal Pengo, il fut accusé d'être possédé par un démon. Nkwera fut excommunié de l'Église en 1999, mais son église, démultipliée par la scission de certains de ses élèves, regroupe de nombreux fidèles. Il n'est pas inintéressant de noter que l'épouse de Nyerere, et certains de ses enfants, sont des fidèles du père Nkwera. Des médias rapportent à ce sujet qu'au cours de la messe funéraire de Nyerere, Maria Nyerere s'agenouilla au moment de la communion - une observance suivie par les fidèles de ministère de Nkwera. Par ailleurs, en Tanzanie, contrairement à d'autres pays africains ${ }^{81}$, les églises historiques n'étaient pas intervenues en faveur de la démocratisation ${ }^{82}$, illustrant la pertinence de l'analyse d'Ali A. Mazrui sur le conservatisme politique du christianisme en Afrique de l'Est ${ }^{83}$. La spécificité du processus de démocratisation politique en Tanzanie n'y est pas étrangère. En effet, l'introduction du multipartisme fut le fait du parti unique - et de Nyerere en premier lieu ${ }^{84}$ - et non de la population qui affirma à une grande majorité son refus du changement lors d'une consultation organisée en 1991 par la commission dite Nyalali ${ }^{85}$. Elle ne fut ni acquise suite à la pression des groupes d'opposition, encore moins à celle de l'Église catholique qui ne joua à cet égard aucune fonction d'éducation politique et civique. Cet ensemble de phénomènes ayant donc conduit d'un côté à une diversification de l'offre religieuse, et de l'autre à une perte de la reconnaissance du rôle des églises historiques, qui n'ont pas joué le rôle d'acteur à part entière de la société civile tanzanienne qu'elle aurait pu avoir si elle s'était impliquée dans l'institutionnalisme du pluralisme politique, contribua à fragiliser la place de l'Église dans l'opinion publique.

Dans le vide idéologique national actuel et en considération de l'incapacité des élites au pouvoir à concrétiser les espoirs suscités par la démocratisation et la libéralisation économique, l'État tanzanien tend à perdre sa légitimité à produire des imaginaires de la nation. De même, le monopole des églises historiques, et de l'Église catholique en premier chef, à l'énonciation d'une vérité religieuse étant concurrencé par les nouvelles églises, c'est son statut d'interlocuteur privilégié au sein de l'État tanzanien qui est remis en cause. Dans un tel contexte, il n'est guère étonnant que chacun de ces deux espaces de pouvoir cherche à retrouver de la crédibilité.

\section{La solution Nyerere?}

20 Parmi les différentes stratégies de récupération d'une légitimité ébranlée qui sont actuellement mises en œuvre, la promotion de la figure revisitée de Nyerere paraitt constituer une ressource symbolique puissante. Sous la forme de "père de la nation " qui diffusa dans la société tanzanienne des valeurs à portée universelle, et contribua à cimenter une nation en déjouant les possibles lignes de fractures religieuses ou ethniques, il est clair que cette figure revisitée par le pouvoir en place permet de 
revivifier les imaginaires de la nation ébranlés par les changements économiques et sociaux récents. À travers l'homme, ce sont les valeurs qui sont brandies : l'unité contre le tribalisme et les clivages religieux, l'honnêteté contre la corruption, la simplicité contre l'accumulation forcenée. La figure du père de la nation contribue par ailleurs à maintenir l'assise populaire du parti au pouvoir. Les politiciens en sont bien conscients, et se rendirent nombreux sur la tombe de l'ancien président pendant les dernières élections présidentielles de fin $2005^{86}$. Au cours de cette même période, alors que certains candidats de l'opposition cherchaient un regain de légitimité en assurant vouloir suivre les pas de l'ancien président Nyerere, le président Mkapa déclara publiquement que seul le CCM, en tant qu'ancien parti unique, pouvait se revendiquer comme le fier héritier de Nyerere ( No one can claim to respect Nyerere more than the Chama Cha Mapinduzi $\left.{ }^{87}\right)$. En rejetant l'usage du symbole Nyerere par les autres partis politiques, le CCM visait donc à se l'approprier. Si le CCM est le maitre d'œuvre de l'usage de la mémoire nyérériste, le nouveau président Jakaya Kikwete se présente officiellement comme le fils héritier de Nyerere. Les références à Nyerere sont redondantes dans nombre de ses discours publics. Les comparaisons incessantes entre celui-ci et le premier président de Tanzanie qui sont diffusées dans les médias relèvent d'une même stratégie d'invention d'une filiation, d'autant que ces comparaisons consistent à ne relever que les similarités entre ces deux hommes. Elles tirent au risible dans certains cas, comme l'exemple qui suit, utilisé de manière récurrente pour asseoir une filiation symbolique : J.K., ce sont bien sûr les initiales de Jakaya Kikwete, mais ce sont aussi celles formées par les deux prénoms de Nyerere, Julius Kambarage. L'apologie du père de la nation glisse, plus ou moins subtilement, vers celle du président Kikwete. En ce sens, l'actuel président est bien l'héritier de Nyerere dans le domaine de l'usage des médias pour la promotion de son image nationale ${ }^{88}$.

21 La demande de canonisation portée devant le Vatican par le clergé de Tanzanie, si elle aboutit, fera basculer l'ancien président dans l'espace du sacré. Sous la forme de "saint» qu'elle prendra alors, cette figure revisitée par l'Église catholique peut contribuer à lui redonner une place d'acteur déterminant du paysage religieux et politique tanzanien. Au plan religieux, l'Église pourrait retrouver une audience nationale large, en offrant la preuve de sa légitimité divine face aux nouvelles églises concurrentes. En effet, la déclaration en sainteté de Nyerere par le Vatican témoignerait de la capacité de l'Église catholique à produire des saints, qu'aucune église nouvelle ne peut aujourd'hui revendiquer. De plus, la possibilité de vénérer Nyerere et de prier pour son intercession pourrait attirer des croyants fréquentant d'autres églises. À cet égard, la canonisation de Nyerere constituerait un pied de nez au Marian Faith Healing Ministry du père Nkwera. Si Julius Nyerere était déclaré un saint catholique, la brèche ouverte dans la crédibilité de l'Église catholique par la fidélité de Maria Nyerere et de certains des enfants de l'ancien président au culte du père Nkwera, qui a contribué à cautionner la légitimité de cette église dans l'espace religieux national et à lui attirer de nouveaux croyants, pourrait être comblée. Au plan politique, la double appartenance de la figure de Nyerere à la sphère politique et à la sphère religieuse qui découlerait d'une déclaration en sainteté par le Vatican pourrait resserrer les liens du catholicisme historique avec le pouvoir politique. On peut dès lors penser que l'inauguration du procès en canonisation est bien un manœuvre stratégique, même si le clergé de Tanzanie insiste sur le fait que la décision de déposer une telle requête auprès du Vatican ne serait pas venue des membres du clergé mais de la population tanzanienne ${ }^{89}$, comme le requiert officiellement la procédure initiale de 
lancement d'une demande en vue d'une investigation en canonisation, basée sur l'existence d'une « réputation de sainteté » au niveau local.

L'usage d'un même outil de légitimation par ces deux espaces de pouvoir que sont l'État et l'Église témoigne du fait que l'Église catholique n'a pas renoncé à toute influence politique, de même que la politique n'a pas renoncé à se servir de la religion. La question de savoir si ces deux sphères agiraient de concert est pertinente. Sachant que d'un côté, la politique en Tanzanie a été si parfaitement dissociée du religieux qu'elle est désormais victime d'un véritable désenchantement allant jusqu'à la diabolisation ${ }^{90}$, et que de l'autre, la religion catholique ne pourra sans doute résister à la concurrence des nouveaux mouvements religieux qu'en s'appuyant sur ses liens avec le monde politique, l'hypothèse d'une manœuvre bicéphale d'idéalisation de Nyerere mérite d'être creusée. Il n'en reste pas moins que le basculement de la figure de Nyerere dans la sphère du divin qu'une canonisation viendrait opérer pourrait mettre le pouvoir politique dans une situation délicate au vu de l'équilibre religieux entre islam et christianisme que, depuis l'indépendance, le pouvoir cherche à construire. Le silence médiatique qui entoure le procès en canonisation pourrait découler du difficile positionnement du monde politique face à une tentative de violation de l'injonction, toujours d'actualité, de ne pas mélanger le politique et le religieux. Les suggestions d'interlocuteurs selon lequel les hommes politiques ne se prononcent pas sur la requête de l'Église parce que justement, en Tanzanie, l'espace du politique et l'espace de religieux sont indépendants, ne tiennent pas devant l'évident chevauchement que, de facto, cette requête impose. La présence d'un président musulman qui, depuis la campagne présidentielle de 2005, sut gérer les tentations de politisation de la religion en ménageant les musulmans et les chrétiens de Tanzanie - les médias suivirent certaines de ses prières à la mosquée, mais ne manquèrent pas de diffuser l'information de sa visite à la veuve du président Nyerere, qui lui remit une Bible, et de sa récente rencontre avec le pape Benoît XVI - peut aussi expliquer le refus de commenter publiquement le procès de canonisation en cours. Sachant le potentiel de violence religieuse qui couve depuis l'introduction du multipartisme, selon de récentes analyses $^{91}$, et notamment à travers l'opposition religieuse et politique entre les îles de Zanzibar et la partie continentale du pays, le silence du pouvoir en place vise à éviter l'introduction, dans la sphère publique, d'une nouvelle cause de conflit politique enracinée dans des ressentiments religieux.

Enfin, il importe de conclure en soulignant que la stratégie de promotion de la figure de Nyerere ne peut fonctionner comme une ressource puissante qu'à la condition que les nouveaux discours qui réinventent le mythe Nyerere soient reçus et réappropriés par la population. Dans l'ensemble, les efforts de création d'un grand récit national assimilé à un seul individu qui ont été entrepris pendant la période socialiste, et leur renforcement par les pratiques autoritaires d'un gouvernement mené par un parti unique puissant, ont porté leurs fruits. La grande popularité du premier président de Tanzanie est un fait incontestable. Pour une majorité de la population, le nom de Nyerere est associé à des qualités de simplicité, de modestie, d'intelligence et d'incorruptibilité qui font défaut à la nouvelle classe politique. Ses accomplissements, principalement une nation soudée et l'absence de conflits religieux ou ethniques, sont sur toutes les lèvres. Ce discours consensuel, que l'école contribue largement à diffuser, est renforcé par la prolifération d'écrits académiques et journalistiques, où toute référence à l'ancien président se fait sur le mode louanger. Pourtant, la présence de voix critiques est un fait à ne pas négliger ${ }^{92}$. Issues de différents espaces, ayant 
différentes légitimités dans l'espace public, ces voix divergentes multiples ont surtout en commun de ne pas être relayées par les médias. Tenues à l'écart des espaces de médiatisation qui pourraient leur donner une visibilité publique, elles peinent à peser sur les représentations de la nation et de l'ancien président Nyerere à l'échelle nationale. La poignée d'anciens hommes politiques ayant directement souffert des pratiques autoritaristes du régime socialiste est notoirement absente de la scène médiatique. Les mémoires anonymes des exactions de l'État, de l'administration publique et de l'armée qui s'entendent au niveau familial, communautaire ou villageois ne sont pas relayées au-delà de leur espace d'expression locale. Parmi les partis politiques d'opposition, seul le Democratic Party du révérend Mtikila propose une alternative critique à l'idéalisation de Nyerere - tous les autres partis d'opposition préférant bénéficier de l'aura nyérériste en cherchant à s'approprier la figure mythifiée de Nyerere. Mais parce que cette alternative critique prend la forme de la calomnie et de la diabolisation, elle est tenue à l'écart des grands circuits de médiatisation. Selon Mtikila"3, son libelle intitulé «Les informations du Parti Démocratique sur les péchés de Julius Kambarage Nyerere ", qui non seulement dénonce les manœuvres frauduleuses et les supercheries de Nyerere mais tente aussi de démontrer que Nyerere était un être mauvais possédé par le désir de faire le mal, ne trouve ni maison d'édition ni imprimerie parce qu'il est censuré par le pouvoir en place. Les quelques cercles intellectuels et politiques critiques semblent aussi touchés par la même impossibilité de s'exprimer publiquement. Ainsi, des journalistes bien en vue semblent craindre de s'attirer les foudres du pouvoir, comme en atteste le commentaire qui m'a été fourni pour expliquer le refus d'un entretien: "My views on and of Nyerere are unprintable and, as such, I had rather keep them to myself. For instance, if I told you that the man is responsible for most of the woes that Tanzanians are in today, you probably would not believe me. Such as institutionalised corruption, unbridled arrogance on the part of the ruling party and its leaders, crushing poverty, etc. ${ }^{94}$ Enfin, même chez les individus acquis au grand récit national nyérériste, la demande de canonisation rencontre le scepticisme. Le Guardian rapporte ainsi cette opinion partagée, à partir de quelques avis glanés à la sortie de la messe dominicale de la cathédrale Saint Peter de Dar es Salaam, selon laquelle Nyerere, aussi pieux et plein de bonnes intentions fut-il, n'en reste pas moins un homme politique qui dut soumettre ses principes humanistes à la raison d'État ${ }^{95}$. La "solution Nyerere " n'est pas gagnée d'avance.

\section{Conclusion}

Il reste finalement à souligner que cette machine à deux moteurs de production de symboles ne manque pas de contradictions, et peut se retourner contre ses créateurs. Les partis d'opposition sont prompts à renvoyer l'exemple idéalisée ${ }^{96}$ de Nyerere à la figure de ses promoteurs au sein du parti en place. Ces derniers bafoueraient sa mémoire, et à travers elle la nation dans son ensemble, en s'adonnant à la corruption, et en négligeant leur devoir de redistribution des richesses publiques.

Ils voueraient un culte hypocrite à ce héros national, louant à voix haute l'héritage moral du socialisme, mais ne se rendant sur sa tombe à Butiama, ou visitant sa veuve, Maria Nyerere, qu'au moment des élections, en vue d'accroître leur popularité. Bref, la figure de Nyerere est utilisée comme un étalon de mesure implacable pour juger les paroles et les actes des politiciens bien en vue. L'État persiste dans sa manœuvre à 
relancer un imaginaire national par la promotion du père de la nation, mais ce faisant, il a tendu une perche aux voix critiques émergentes pour mieux le flageller. S'il a tout à gagner d'une unité nationale renforcée, chacun de ses membres pris à part en mesure les conséquences. Quant au procès en canonisation, dont le secret qui l'entoure semble bien avoir pour objectif d'éviter qu'un échec à démontrer les vertus chrétiennes dont aurait fait preuve Nyerere ne vienne porter un coup fatal à la crédibilité de l'Église de Tanzanie, mais aussi d'éviter les accusations que le parti en place ne respecterait pas l'équilibre interreligieux et la séparation du politique et de la religion au fondement de la culture politique tanzanienne, il semble avoir été reçu avec beaucoup de scepticisme par la population. On peut se demander si cette étape dans la sanctification du père de la nation ne va pas agir de manière contraire à ses objectifs : au lieu de parachever le processus de mythification de Nyerere en mettant une cerise religieuse sur le gâteau nationaliste, il pourrait abattre le château de cartes fragile en ces temps d'incertitudes pour la population.

\section{BIBLIOGRAPHIE}

Anderson, B. (1991) [1983]. Imagined Communities. Reflections on the Origin and Spread of Nationalism. London : Verso.

Amitiés franco-tanzaniennes (1981). La Tanzanie : Vers un autre développement ? Paris : Amitiés franco-tanzaniennes .

Amitiés franco-tanzaniennes (1983). La Tanzanie, espoirs et réalités. Paris : Amitiés francotanzaniennes.

Askew, K.M. (2002). Performing the Nation: Swahili Music and Cultural Politics in Tanzania. Chicago : University of Chicago Press.

Askew, K.M. (2006). « Sung and Unsung: Musical Reflections on Tanzanian Postsocialisms. » Africa 76 (1) : 15-43.

Assensoh, A.B. (1998). African Political Leadership, Jomo Kenyatta, Kwame Nkrumah, and Julius K. Nyerere. New York : Krieger Publishing Co.

Balandier, G. (1969). Anthropologie politique. Paris : PUF.

Balandier, G. (1980). Le pouvoir sur scène. Paris : Balland.

Baregu, M. (1994). « The Rise and Fall of the One-Party State in Tanzania. » In J.A. Widner (ed.), Economic Change and Political Liberalization in Africa: 156-181. Baltimore : Johns Hopkins University Press.

Bayart, J.-F. (1993). Religion et modernité politique en Afrique Noire. Dieu pour tous et chacun pour soi. Paris : Karthala.

Blommaert, J. (1999). State Ideology and Language in Tanzania. Koln : Koppe.

Booth, D. (1994). Social, Economic and Cultural Change in Contemporary Tanzania. A People-Oriented Focus. Stockholm : SIDA. 
Bourdieu, P. (1986). «L'illusion biographique. » Actes de la recherche en sciences sociales n 62-63. Centlivres, P. (ed.) (2001). Saints, Sainteté, et Martyrs. La Fabrication de l'exemplarité. Paris : Edition de l'Institut d'Ethnologie de Neuchâtel \& Editions de la Maison des Sciences de l'Homme.

Charuty, G. (1995). « Logiques sociales, savoirs techniques, logiques rituelles. » Terrain (« La fabrication des saints »), $24: 5-14$.

Civille, J.R., Duggan, R. (1976). Tanzania and Nyerere: A Study of Ujamaa and Nationhood. Maryknoll : Orbis Books.

Comoro, C., Sivalon, J. (1999). « The Marian Faith Healing Ministry. An African Expression of Popular Catholicism in Tanzania. » In T. Spear, I.N. Kimambo (ed.), East African Expression of Christianity : 275-295. Oxford et al. : James Currey.

Condon, J.C. (1967). « Nation Building and Image Building in the Tanzanian Press. » The Journal of Modern African Studies 5 (3) : 335-54.

Constantin, F., Coulon, C. (dir.) (1997). Religion et transition démocratique en Afrique. Paris : Karthala. Constantin, F., Lafargue, J. (1996). «Tanzanie: Elections pluralistes. Désordre, continuité, changement ?. » L'Afrique Politique 1996 : Démocratisation: Arrêt sur images : 145-171. Paris : Karthala. Constantin, F., Martin, D.-C. (1988). Arusha (Tanzanie). Vingt ans après. Pau : Université de Pau et des Pays de l'Adour.

Corten, A., Mary, A. (2000). Imaginaires politiques et pentecôtismes. Paris : Karthala.

Crozon, A. (1998). « Dire pour séduire : langages et politique en Tanzanie. » In D.-C. Martin (dir.), Nouveaux langages du politique en Afrique Orientale : 115-185. Paris, Nairobi : Karthala, IFRA.

Dumont, R. (1962). L'Afrique noire est mal partie. Paris : Le Seuil.

Edmondson, L. (2007). Performance and Politics in Tanzania. The Nation on Stage. Bloomington and Indianapolis : Indiana University Press.

Fair, L. (2001). Pastimes and Politics. Culture, Communication, and Identity in Post-Abolition Urban Zanzibar, 1890-1950. Athens : Ohio University Press.

Fouéré, M.-A. (2008a). «J.K. Nyerere entre mythe et histoire : analyse de la production d'une mémoire officielle en Tanzanie contemporaine. Les commémorations de la disparition du père de la nation. » Cahiers d'études africaine (à paraître).

Fouéré, M.-A. (2008b). « Scandales financiers et remous politiques en Tanzanie. » Mambo! La lettre d'information de l'Institut français de recherche en Afrique VII (4).

Fouéré, M.-A. (2007). « Nyerere, Julius Kambarage. » In L.L. Kaid, C. Holtz-Bacha, Encyclopedia of Political Communication : 517-518. Thousand Oaks : SAGE Publications.

Fouéré, M.-A. (2006). « The Legacy of J.K. Nyerere and the Tanzanian Elections of Oct.-Dec. 2005. » In H. Charton, C. Médard (ed.), Annuaire de l'Afrique Orientale 2005. Nairobi : IFRA.

Forster, P. (1987). « Religion and the State in Tanzania and Malawi. » Journal of African and Asian Studies XXXII (3-4) : 163-84.

Geiger, S. (1997). TANU Women: Gender and Culture in the Making of Tanganyika Nationalism, 1955-1965. Portsmouth (NH) : Heinemann.

Joinet, B. (1977). Le soleil de Dieu en Tanzanie. Paris : Editions du Cerf.

Joinet, B. (1981). Tanzanie : Manger d'abord. Paris : Karthala. 
Hansen, H.B., Twaddle, M. (ed.) (1995). Religion and Politics in East Africa. London : James Currey ; Nairobi : East African Educational Publishers ; Kampala : Fountain Publishers; Athens (OH) : Ohio University Press.

Harries, L. (1969). « Language Policy in Tanzania. » Africa 39 (3) : 275-80.

Hatch, J. (1976). Two African Statesmen: Kaunda of Zambia and Nyerere of Tanzania. London : Secker \& Warburg.

Heilman, B., Kaiser, P. (2002). « Religion, Identity and Politics in Tanzania. » Third World Quarterly. 23 (4) : 691-709.

Huddleston, T.R. (1995). « The person Nyerere. » In C. Legum, G. Mnari (eds.), Mwalimu, the Influence of Nyerere : 1-8. London : James Currey.

Hyden, G. (1980). Beyond Ujamaa in Tanzania: Underdevelopment and an Uncaptured Peasantry. London, Ibadan, Nairobi : Heinemann.

Katare, E. (2007). Mwalimu Julius Kambarage Nyerere, Falsafa na Dhana ya Utakatifu. Dar es Salaam : Creative Print [Mwalimu Julius Kambarage Nyerere, Philosophie et concept de canonisation].

Kaiser, P. (1996). «Structural Adjustment and the Fragile Nation. The Demise of Social Unity in Tanzania. » Journal of Modern African Studies 34 (2) : 227-37.

Kelsall, T. (2003). « Governance, Democracy and Recent Political Struggles in Mainland Tanzania. » Commonwealth \& Comparative Politics 41 (2) : 55-82.

Kidanka, C. (2006). " The state of the media in Tanzania. » A paper presented at the Journalists' Conference for the East and Horn of Africa, 27-29 November, Entebbe, Uganda.

Kihl, L. (2006). Religion et politique : Les organisations religieuses, de nouveaux acteurs politiques? Mémoire de Master, Université Paris 1 Panthéon-Sorbonne.

Konde, H.S. (1984). Press Freedom in Tanzania, Arusha. Eastern Africa Publications : Lederbogen, Utz.

Legum, C., Mnari G. (1995). Mwalimu, the Influence of Nyerere. London : James Currey.

Liviga, A. (1999). « Ujamaa, le poids d'un legs. » In C. Baroin, F. Constantin, La Tanzanie contemporaine : 67-76. Paris : Karthala-IFRA.

Ludwig, F. (1996). « After Ujamaa. Is Religious Revivalism a Threat to Tanzania's Stability? » In D. Westerlund (ed.), Questioning the Secular State, The Worldwide Resurgence of Religion in Politics. London : Hurst \& Company.

Ludwig, F. (1999). Church and State in Tanzania, Aspects of a Changing Relationships, 1961-1994. LeidenBoston-Köln : Brill.

Martin, D.-C. (1988). Tanzanie : L'invention d'une culture politique. Paris : Presse de la Fondation nationale des sciences politiques-Karthala.

Martin, D.-C. (1993). « La Tanzanie et le multipartisme. » Afrique Contemporaine 167 : 3-13.

Martin, D.-C. (1998). (dir.) Nouveaux langages du politique en Afrique Orientale. Paris : Karthala-IFRA.

Maw, J., Parkin, D. (1985). Swahili Language and Society. Vienne : Beitrage für Afrikanistik.

Maw, J., Omar, A. (1985). « The Language of Social Control. » In J. Maw, D. Parkin, Swahili Language and Society : 151-158. Vienne : Beitrage für Afrikanistik.

Mazrui, A. (1967). « Tanzaphilia : a Diagnosis. » Transitions 31 : 20-26. 
Mazrui, A. (1973). « The Sacred and the Secular in East African Politics. » Cahiers d'Études Africaines $13(4): 664-681$.

Mazrui, A. (2002). The Titan of Tanzania: Julius K. Nyerere's Legacy. Binghamton : The Institute of Global Cultural Studies.

Mbise, I. (1989). « L'invention des symboles d'une éthique nationale. » In D.-C. Martin, H. Batibo, Tanzanie. L'Ujamaa face aux réalités : 81-85. Paris : Éditions Recherche sur les Civilisations.

McHenry, D.E. (1994). Limited Choices: The Political Struggle for Socialism in Tanzania. Boulder, Colorado : Lynne Rienner.

Mmuya, M., Chaliha, A. (1992). Towards Multi-Party Democracy in Tanzania. Dar es Salaam : Dar es Salaam University Press.

Mtikila, Rev. C. (2006). Taarifa ya Democratic Party juu ya madhambi ya Julius Kambarage Nyerere, non publié [Les informations du Parti Démocratique sur les péchés de Julius Kambarage Nyerere].

Mytton, G. (1976). The Role of the Mass Media in Nation-Building in Tanzania, PhD Thesis, Manchester University.

Nkwera, F. (2005). Exorcism vs. Demons' Strategies. Dar es Salaam : Marian Faith Healing Ministry.

Nora, P. (1984-1986). Les lieux de mémoire, I, II, III. Paris : Gallimard.

Nyerere, J.K. (1963). Juliasi Kaisari. Nairobi, Dar es Salaam : Oxford University Press.

Nyerere, J.K. (1967). Freedom and Unity, Uhuru na Umoja. A selection from writings and speeches 1952-1965. London : Oxford University Press.

Nyerere, J.K. (1968). Ujamaa. Essays on Socialism. Dar es Salaam : Oxford University Press.

Nyerere, J.K. (1969). Mabepari wa Venisi. Dar es Salaam : Oxford University Press.

Nyerere, J.K. (1973). Freedom and Development, Uhuru na Maendeleo. Dar es Salaam : Oxford University Press.

Nyerere, J.K. (1974). Man and Development. Dar es Salaam, Oxford University Press.

Nyerere, J.K. (1977). The Arusha Declaration ten years after. Dar es Salaam : Government Printer.

Okema, M. (1996). Political Culture of Tanzania. Lewiston : Edwin Mellen Press.

Othman, H. (1999). « Nyerere, le premier Tanzanien. » In C. Baroin, F. Constantin, La Tanzanie contemporaine : 59-66. Paris : Karthala-IFRA.

Othman, H. (2007). « Mwalimu Julius Nyerere. An Intellectual in Power. » In H. Othman (ed.), Sites of Memory. Julius Nyerere and the Liberation Struggle of Southern Africa. Dar es Salaam : Zanzibar International Film Festival-ZIFF.

Ovchinnikov, V. (2005). « Mwalimu Julius Kambarage Nyerere (1922-1999). Teacher, Politician, Poet. » In RASIAS, Russian Academy of Sciences Institute for African Studies, Julius Nyerere. Humanist, Politician, Thinker : 56-62. Dar es Salaam : Mkuki na Nyota [ $1{ }^{\text {re }}$ édition en 2003, Julius Nyerere: Humanist, Politician, Thinker, Peramiho, Benedictine Publication Ndanda].

Ranger, T. (1975). Dance and Society in Eastern Africa, 1890-1970: The Beni Ngoma. Berkeley : University of California Press.

Ricard, A. (1998). Ebrahim Hussein, Théâtre swahili et nationalisme tanzanien. Paris : Karthala.

Ricard, A., Garnier, X. (ed.) (2003). « Littérature swahili. » Numéro spécial, 16, Etudes littéraires africaines, Paris. 
Shivji, I.G. (1992). « The politics of Liberalization in Tanzania : The crisis of Ideological Hegemony. » In H. Campell, H. Stein (ed.), Tanzania and the FMI. The Dynamics of Liberalization. Boulder : Westview Press.

Smith, W.E. (1972). We Must Run While They Walk: A Portrait of Africa's Julius Nyerere (aussi publié sous le titre Nyerere of Tanzania, 1973, reissued 1981).

Spear, T., Kimambo, I.N. (ed.) East African Expression of Christianity. Oxford et al. : James Currey. Stoger-Eising, V. (2000). « Ujamaa revisited: Indigenous and European influences in Nyerere's Social and Political Thought. » Africa 70 (1): 118-143.

Thiesse, A.-M. (1999). La création des identités nationales, Europe $\mathrm{XIII}^{e}-\mathrm{XX}$ siècle. Paris : Seuil.

Toulabor, C. (1997). « Quand le diable lit la Bible : nouvelles Églises, modernité et socialisation à Accra. » In F. Constantin, C. Coulon (dir.), Religion et transition démocratique en Afrique. Paris : Karthala.

Urfer, S. (1971). Ujamaa, espoir du socialisme africain en Tanzanie. Paris : Aubier.

Urfer, S. (1975). Socialisme et Église en Tanzanie. Paris : IDOC-France.

Urfer, S. (1974). Une Afrique socialiste : La Tanzanie. Paris : Editions Ouvrières.

Van Bergen, J.P. (1981). Development and Religion in Tanzania. Sociological Soundings on Christian Participation in Rural Transformation. Madras : IIMO ; Leiden : Christian Literature Society.

Westerlund, D. (1980). Ujamaa na Dini: A Study of Some Aspects of Society and Religion in Tanzania, 1961-1977. Stockholm : Borgstroms Tryckeri.

Westerlund, D. (1980). « Christianity and religion in Tanzania, 1967-1977. » Journal of Religion in Africa 11 (1) : 30-55.

Whiteley, W.H. (1969). Swahili. The Rise of a National Language. London : Methuen.

Yahya-Othman, S. (1999). « Kiswahili, la consécration d'une langue nationale africaine. » In C. Baroin, F. Constantin, La Tanzanie contemporaine : 77-88. Paris : Karthala-IFRA.

\section{Journaux}

Citizen (The), 14/10/2008, « Mwalimu Nyerere's rich legacy. Church taking first steps to declaring him a saint. "

Guardian (The), 05/03/2007, « Tanzanians wonders whether Nyerere saint or sinner. » Guardian (The), 24/01/2006, « Is Nyerere process to sainthood timely? » Guardian (The), 14/10/2005, « Lengthy process to Nyerere's sainthood. » Majira, 05/12/2005, « CCM yaonywa Mwalimu Nyerere si mali ya mtu. » Mtanzania, 21/10/2005, « Mkapa awashambulia wapinzani. » New African (The), 2006, « Arise Saint Nyerere? » Novembre. Nipashe, 05/03/2007, « Mwl. Nyerere na mchakato wa kuwa mwenye heri. » Nipashe, 05/10/2005, « Dua kwenye kaburi la Nyerere wakati huu kuna maana gani? » Patriotic Vanguard (The), 10/03/2007, « Tanzania's Nyerere to be canonised. » Standard (The), 08/04/2006, « Why Catholics want Nyerere canonised. » 
Sunday Citizen, 14/10/2007, « Mwalimu's canonisation: How tenable is the process?» Sunday News, 14/10/2007» Justified case for beatification. » Nyerere Special Supplement. Young African, 26/09/2006, « Tanzania's first president to become a Saint? »

\section{Sites internet}

www.dacb.org/stories/tanzania/nyerere.html: « Julius Kambarage Nyerere, 1922 to 1999, Roman Catholic Church, Tanzania. » Dictionary of African Christians, par le Révérend Angolowisye Isakwisa Malambugi.

theworldimho.blogspot.com /2006/08/ julius-nyerere-likely-to-be-bestowed.html: « Julius Nyerere likely to be bestowed sainthood » $(25 / 08 / 2006)$.

http://www.cwnews.com/news/viewstory.cfm?recnum=42139: « Beatification inquiry for Tanzania's Nyerere. » Catholic World News (30/01/2006).

http: / /halfricanrevolution.blogspot.com/2007/03/nyerere-saint-or-sinner.html: « Nyerere : Saint or Sinner?»(06/03/2007).

http://www.thepatrioticvanguard.com/article.php3?id article=1082: « Tanzania's Nyerere to be canonized. » The Patriotic Vanguard, par Patrick Hassan-Morlai (10/03/2007).

http://www.stanleymeisler.com/news-commentary/nyerere.html: « Saints and Presidents. A commentary on Julius Nyerere » par Stanley Meister (17/12/2006).

eadstotanzania.typepad.com/eadstotanzania/julius nyerere/: « Julius Nyerere » par Daniel Eads (15/01/2008; 12/01/2008, 11/01/2008, 10/01/2008, 09/01/2008).

\section{ANNEXES}

\section{Prière de demande en grâce pour l'intercession du serviteur de Dieu, Julius Kambarage Nyerere}

Introduite par le diocèse de Musoma, Tanzanie.

o God our Father, you have created us so that we may know you, serve and love you and love our fellow sisters and brothers.

We thank you, O God our Creator, for the gift of your servant, Julius Kambarage Nyerere-a lay faithful and a father of the family-who led your people of Tanzania as true father and teacher.

Our Father, your servant gave himself up completely for your people with love that knew no boundaries, building tirelessly unity and solidarity among all Children of God. He cared for the poor. He uplifted the downtrodden. He consoled orphans. He welcomed and fed refugees.

o God, your servant beingfaithful to you and your Church-has become for us a true example of deep faith and genuine piety. He cherished a great love and respect for the Virgin Mary, the Mother of your Son and our Lord Jesus Christ. He dedicated the People of Tanzania to Our Lady so that they may live peacefully under her maternal protection. 
o God, your servant Julius Kambarage Nyerere showed great zeal in teaching everyone to know and honour you. He defended justice and fought relentlessly against oppression and discrimination of any kind. His humble service for all people remains for all generations an example to imitate on their way to you. Almighty Father, grant us, by his intercession, and according to your will, the graces we implore. May his holiness become evident to all People of God so that he will soon be numbered among your saints. Amen.

O Dieu notre père, tu nous as créés pour que nous te connaissions, te servions et te chérissions, et chérissions nos frères et sœurs.

Nous te remercions, O Dieu notre Créateur, pour le cadeau de ton serviteur, Julius Kambarage Nyerere - un fidèle croyant et un père de famille - qui a conduit ton peuple de Tanzanie comme un vrai père et comme un maître d'école.

Notre père, ton serviteur s'est totalement donné pour ton peuple avec un amour sans bornes, construisant inlassablement l'unité et la solidarité parmi tous les Enfants de Dieu. Il s'est occupé des pauvres. Il relevait les opprimés. Il consolait les orphelins. Il accueillait et nourrissait les réfugiés.

O Dieu, ton serviteur fidèle, à ton Église et à toi-même, est devenu pour nous un vrai exemple de foi profonde et de piété authentique. Il avait un amour sincère et un respect pour la Vierge Marie, la mère de votre fils et du seigneur Jésus Christ. Il a dédié le peuple de Tanzanie à notre Mère pour qu'il vive en paix sous sa protection maternelle.

O Dieu, ton serviteur Julius Kambarage Nyerere a témoigné d'un grand zèle en enseignant à tous comment te connaître et t'honorer. Il défendait implacablement la justice et combattait sans relâche l'oppression et les discriminations de toute sorte. Son service humble pour tous reste pour toutes les générations un exemple à imiter dans le chemin qui conduit vers toi.

Dieu tout-puissant, accorde-nous, par son intercession, et selon ta volonté, les grâces que nous implorons. Que sa sainteté éclate aux yeux du peuple du Dieu afin qu'il compte bientôt parmi tes saints. Amen.

(traduction de Marie-Aude Fouéré)

\section{NOTES}

1. Dans le christianisme, la canonisation est l'acte par lequel le Pape attribue à un individu le titre de "saint ", l'inscrivant sur la liste officielle des saints reconnus par l'Église catholique. Tout procès en canonisation s'opère suivant quatre étapes: l'attribution du statut de "serviteur de Dieu ", de "vénérable ", de "bienheureux " (suite à une béatification) et enfin de "saint». La suite du présent texte présente ces diverses démarches en détail.

2. Certaines biographiques indiquent que Butiama est le village natal de Nyerere, d'autres qu'il s'agit d'un village proche de son lieu de naissance, et où il ne se rendit pour la première fois qu'à son entrée en école primaire.

3. L'archevêque du diocèse de Mwanza depuis 1987, Anthony Mayala, prit formellement la suite de la supervision du procès en canonisation de Nyerere après le décès de 
l'évêque Justin Samba le 23 août 2006. Toutefois, le suivi revient aujourd'hui à l'évêque Michael G.M. Msonganzila, nommé au diocèse de Musoma à la fin 2007.

4. Selon les biographes, l'année du mariage de Nyerere est soit 1952, soit 1953.

5. La tâche du postulateur, nommé par l'évêque du diocèse, consiste à assurer l'instruction préalable du dossier et à défendre le candidat en sainteté. C'est lui qui, pendant l'enquête en sainteté, opère la sélection des témoins à interroger pour recueillir des preuves sur les vertus du candidat en sainteté. Une fois l'enquête achevée, il porte la cause au Vatican où il réside pendant toute la durée de la rédaction du document final (la Positio) qui sera étudié par la congrégation des cardinaux pour la cause des saints. Il est aidé dans sa tâche d'un vice-postulateur.

6. La Société de missions étrangères catholiques d'Amérique, encore appelée Maryknoll, fut fondée en 1911 par les évêques catholiques des États-Unis d'Amérique. Elle relève des ordres dominicains. Elle envoie des missionnaires en Afrique, en Asie et sur le continent américain. En Tanzanie, la société Maryknoll soutient le diocèse de Musoma depuis 1946 après que quatre pères s'installent dans la région. C'est un des quatre fondateurs de cette mission, le père William Collins, qui célébra le mariage Nyerere. La société soutint financièrement certains des déplacements internationaux de Nyerere, notamment en 1956, lorsque Nyerere se rendit aux Nations Unis à New York pour plaider l'indépendance de son pays, alors nommé Tanganyika.

7. Civille et Duggan (1976). L'ouvrage est paru chez Orbis Book, la maison d'édition de la société Maryknoll.

8. Les censeurs théologiques ont pour charge d'examiner les écrits laissés par le candidat en canonisation avant l'introduction du procès, pour déterminer leur valeur spirituelle.

9. Fait archevêque du diocèse de Dar es Salaam en 1992, Polycap Pengo en fut nommé le cardinal en 1998.

10. Voir le titre Young African (26/09/2006): «We are presenting his cause based on his life as a Christian and how his faith influenced his entire political career " (Nous présentons sa cause sur la base de sa vie de chrétien, et sur la manière dont sa foi influença toute sa carrière politique).

11. La dernière réforme du droit en canonisation fut apportée par le pape Jean-Paul II par la Constitution apostolique du 25 janvier 1983 (Divinus Perfectionis Magister), destinée à simplifier la procédure et à y associer plus étroitement les évêques. Elle diminuait aussi l'importance des miracles dans l'attribution du statut de saint, portant l'attention sur la sainteté de la vie menée. Elle renforçait l'autorité du pape en lui laissant la décision finale. L'objectif de cette réforme consistait à promouvoir les béatifications et les canonisations à grande échelle, dans le cadre de la stratégie mondiale pour une nouvelle évangélisation, lancée par Jean-Paul II. Au cours de ses 21 années de pontificat, ce dernier opéra 820 béatifications et 285 canonisations.

12. La Positio se compose de quatre parties : l'informatio présente l'histoire de la cause, les sources, la renommée de sainteté du candidat, et les avis des censeurs théologiques ; le Summarium reprend les témoignages et les documents importants, pour soutenir la Cause ; la Biographia documentata reconstitue la vie du candidat; et le Studium criticum super heroicitate virtutum examine scientifiquement les vertus religieuses et morales dont le candidat fit preuve de son vivant. 
13. Voir le magazine catholique basé à Londres Mission Today (2007): «While we are doing all we can do to aid the canonisation process, we already believe Julius Nyerere is a saint " (Pendant que nous faisons tout notre possible pour aider le procès en canonisation, nous croyons déjà que Julius Nyerere est un saint).

14. Voir «la prière de demande en grâce pour l'intercession du serviteur de Dieu » retranscrite en fin d'article.

15. Citizen (14/10/2008).

16. « He did not kill his political enemies (...) as many of his contemporaries did.»

17. "All those who have information about him both positive and negative, let them bring it to the nearest Cathoclic Church » (Tous ceux qui détiennent des informations sur lui, qu'elles soient positives ou négatives, sont appelés à aller témoigner à l'église la plus proche).

18. On revient sur les raisons du silence du monde politique tanzanien, et des médias, dans la suite du texte.

19. Le Daily News est propriété du groupe paraétatique TSN (Tanzania Standard Newspaper). The Guardian et Nipashe sont des quotidiens publiés par le groupe privé IPP Media, qui regroupe 9 journaux, 4 chaînes de télévision, et 2 stations de radios. IPP Media est détenu par un homme d'affaire proche du pouvoir, Reginald A. Mengi, ancien président du National Board of Business Accountants and Auditors et actuel président de la Confederation of Tanzania Industries (CTI).

20. Voir la bibliographie en fin d'article.

21. L'année de baptême de Nyerere varie selon les auteurs ; 1942 chez Assensoh (1998, p.119), 1943 chez Westerlund ( $1980: 78)$.

22. Ludwig (1999: 78).

23. Ludwig (1999 : 78), Hatch (1976: 6, 236), Civille \& Duggan (1976: 43).

24. www.maryknollafrica.org/Tanzania.htm [archive: https://web.archive.org/web/ 20150104144819/www.maryknollafrica.org/Tanzania.htm].

25. Aussi mentionné par Ludwig (1999 : 80 note 5), qui rencontra le père Walsh en 1977 - ce dernier lui confirma la véracité de ce fait.

26. Hatch (1976) cité dans Ludwig (1999: 78) mais sans donner la page. «Il n’a jamais seulement été un membre nominal de l'Église. Il a toujours mis en pratique sa religion et suivi ses observances ".

27. Laurian Rugambwa, premier évêque tanzanien de Tanzanie en 1952, fut aussi le premier cardinal africain de l'Église catholique, nommé par le Pape Jean XXIII. Archevêque du diocèse de Dar es Salaam de 1968 à 1992, il laissa sa place au cardinal Polycarp Pengo à sa retraite, à l'age de 80 ans. Il est décédé en 1997. Une requête pour sa canonisation est actuellement envisagée.

28. Cité, notamment, dans Sunday Citizen (14/10/2007) : «Sans une messe quotidienne, il m'aurait été impossible de faire mon travail ».

29. Ovchinnikov (2005). Nyerere traduisit des passages de la Bible dans la langue locale des Zanaki of the Bible into Zanaki, et écrivit des poèmes et des chants liturgiques inspirés des évangiles des Apôtres.

30. Voir notamment Katare (2007).

31. Bourdieu (1986). 
32. La suite de l'article revient en détail sur la question de l'idéologie socialiste et de ses ancrages humanistes.

33. Sur ces thèmes, voir Fouéré (2006, 2008). Voir aussi l'analyse d'Askew (2006) sur les chants de lamentations à la gloire de Nyerere qui se sont multipliés après son décès.

34. Les titres de certains des écrits de Nyerere en témoignent : Freedom and Unity, Uhuru na Umoja (1967) - Liberté et unité ; Freedom and Development, Uhuru na maendeleo (1973) Liberté et développement.

35. Je renvoie ici à la remarque de D.-C. Martin (1988: 90) selon laquelle Nyerere, suivant une expression qu'il s'attribuait lui-même, était un "idéaliste-réaliste ", soucieux des principes mais capable de les ajuster au contexte économique, politique et social.

36. Mazrui (2002).

37. Sur les politiques de construction des imaginaires de la nation, voir notamment les analyses classiques de Anderson (1985), Nora (1984-1986) ou encore Thiesse (1999).

38. Un article paru à l'occasion du premier anniversaire de la disparition de Nyerere se donne pour ligne directrice une comparaison entre l'ancien président et le prophète Moïse (The Guardian, 24/10/2000).

39. La République Unie de Tanzanie naquit le 29 octobre 1964 de l'union de le République du Tanganyika (qui avait accédé à l'indépendance en décembre 1962) et de l'ancien sultanat de Zanzibar (devenu indépendant en janvier 1964 sous l'appellation de République Populaire de Zanzibar et Pemba).

40. Selon le titre de l'ouvrage classique de Martin (1988).

41. Ce point est détaillé dans la partie suivante sur les rapports entre l'État et l'Église pendant la période socialiste, entre 1967 et 1985.

42. Parmi de nombreux travaux sur le swahili comme ressource politique, voir notamment Blommaert (1999), Harries (1969), Yahya-Othman (1999), Maw \& Parkin (1985), ou encore Whiteley (1969).

43. Sur les differents registres qui ont influencé la pensée de Nyerere, voir StogerEismg (2000).

44. Voir notamment Ranger (1975), Geiger (1997), Askew (2002), et Edmondson (2007).

45. On ne revient pas ici sur le succès du socialisme à l'africaine de Nyerere sur la scène internationale - avant la découverte des échecs économiques qu'elle produisit - et qui fit dire à des observateurs comme Ali Mazrui (1967) qu'une vraie «tanzaphilie » s'était développée dans les milieux académiques des pays occidentaux dans les années 1960 et 1970. Pour la France, voir ce qu'en dit Amitiés Franco-Tanzaniennes (1983 : 59), à savoir qu' "analysée comme la possibilité d'une troisième voie, d'un socialisme empreint de l'humanisme chrétien de Nyerere, "l'expérience tanzanienne" rencontrait les aspirations de divers milieux français ». Les ouvrages de Louis Dumont (1962), de Sylvain Urfer (1971, 1974) ou de Bernard Joinet $(1977,1981)$ témoignent aussi de l'espoir mis dans l'expérience nyérériste.

46. Nyerere $(1967,1968,1973)$. Voir le titre de l'article de Othman (2007), «Un Intellectuel au pouvoir ".

47. Voir La déclaration d'Arusha vint ans après, ouvrage dans lequel Nyerere fait part de ses analyses désabusées sur l'expérience socialiste, ses réussites et ses échecs (Nyerere, 1977). 
48. Ricard (1998), Ricard et Garnier (2003).

49. Nyerere $(1963,1969)$.

50. Voir Condon (1967), Fouéré (2007), Konde (1984), Mytton (1976).

51. Cité dans Konde (1984). "Le nouveau Standard sera un journal socialiste. Il soutiendra l'idéologie socialiste de Tanzanie telle qu'elle est définie dans la Déclaration d'Arusha ».

52. Martin (1988 : 104-105, 144-143), Konde (1984).

53. Martin (1988) : 145.

54. Certaines restrictions à la liberté de religion sont notables, comme le note Westerlund (1980a: 62). Il s'est agi notamment de l'interdiction d'organisations ouvertement opposée à l'Ujamaa, comme les Témoins de Jéhovah, ou certains aspects des religieux traditionnelles, comme la sorcellerie.

55. Cité dans Westerlund (1980a : 57). «L'histoire a montré combien il est désastreux de mélanger politique et religion. (...) C'est pour cela qu'il est impératif que la religion soit tenue à l'écart de la vie politique de notre pays. "

56. Westerlund (1980a: 63).

57. L'expression est empruntée au titre de la contribution de Mbise (1989) sur le symbolisme socialiste en Tanzanie.

58. "Nationalism is mixed with religious elements which are acceptable to Muslims as well as to Christians, and partly also to adherents of African religion » (Westerlund, 1980a: 68).

59. Westerlund (1980a).

60. Constantin \& Coulon (1997:12).

61. Ainsi, la cérémonie d'investiture de Jakaya Mrisho Kikwete à la présidence du pays en 2005 a débuté par une prière des représentants des différentes religions. La sécheresse qui a touché la Tanzanie en mars 2006 a également conduit les dirigeants politiques à demander à tous les responsables religieux à travers le pays de prier pour qu'il pleuve (Kihl, $2006: 28)$.

62. Urfer (1971: 177).

63. Westerlund (1980a) : 58.

64. Nyerere (1974: 98): "Only by activities in these fields can the church justify its relevance in the modern world. For the purpose of the church is Man-his human dignity and his right to develop himself in freedom. For all human institutions including the church, are established in order to serve man. And it is the institution of the church, through its members, which should be leading to attack on any organization, or any economic, social, or political structure which oppresses men, and which denies to them the right and power to live as the sons of a loving God " (C'est seulement par ses activités dans ces domaines que l'église peut justifier de sa pertinence dans le monde moderne. Car l'objectif de l'église est l'homme - sa dignité humaine et son droit à se développer dans la liberté. Car toutes les institutions humaines, l'église incluse, sont établies pour servir l'homme. Et c'est l'institution de l'église qui, à travers ses membres, devrait être leader dans l'attaque de toute organisation, ou de toute structure économique, politique, sociale qui oppresse les hommes, et qui leur dénie le droit et le pouvoir de vivre comme les fils d'un Dieu d'amour). 
65. Dans des discussions avec des supérieurs catholiques au palais présidentiel, Nyerere aurait déclaré : « Je serais très heureux si les séminaires pouvaient produire des prêtres socialistes » ("I should be very happy if the Seminaries could turn out socialist priests »). Cité dans Westerlund (1980a : 59).

66. Urfer (1971: 177).

67. Kihl (2006:25).

68. Un ministre du culte luthérien, T. Mussa, membre du comité central du parti unique aurait émis l'idée que «quand le christianisme est enseigné dans toute son authenticité, il est exprimé en termes socialistes » ("When Christianity is truly taught it is expressed in socialistic terms »), cité dans Westerlund (1980a: 59).

69. Ludwig (1996: 221).

70. Nyerere aurait confié à l'évêque Robert Rweyemamu, alors secrétaire général de la Tanzania Episcopal Conference (TEC), qu'il avait délibérément décidé de nommer un Chrétien à la tête du département d'éducation politique du parti unique. Sur des confidences similaires de Nyerere à des membres du clergé, voir aussi Ludwig (1996.), Smith (1972), Westerlund (1980a), et Van Bergen (1981).

71. Balandier (1969: 118).

72. Comme le souligne Martin (1988:286-289), l'amorce des changements politiques et économiques qui vont gagner en ampleur en 1985 se situe dès la fin des années 1970, avec l'encouragement de l'initiative privée et le retour d'entreprises nationalisées au secteur privé dès 1977, l'adoption du programme national de survie économique (National Economic Survival Programme) et la réorganisation du parti unique en 1982, ainsi que rétablissement des conseils de district et de coopératives, la refonte de la politique agricole en 1983, et l'adoption d'une Constitution de la République qui limite les mandats du président et augmente le nombre de députés élus. Bref, de nombreux ajustements économiques et politiques précédèrent et annoncèrent le tournant de l'année 1985.

73. Booth (1994).

74. Fouéré (2008).

75. Crozon (1998).

76. Liviga (1999 : 68). Voir aussi l'analyse de Crozon (1998) sur les nouveaux langages du politique retraçant l'émergence, depuis la libéralisation économique et la démocratisation politique, d'un nouveau vocabulaire politique, qui tente de remodeler les imaginaires de la nation, et pallier ainsi le vide laissé par l'idéologie ujamaa.

77. Parmi les très nombreux écrits sur le pentecôtisme, voir notamment Bayart (1993), et Corten \& Mary (2000).

78. Toulabor (1997).

79. Comoro and Sivalon (1999).

80. L'Église doit être détruite par tous les moyens. Il convient de commencer au sommet. Nous devons la détruire pour accéder au superpouvoir de l'humanité ", tiré de Nkwera (2005).

81. Comme le rappelle Lorraine Kihl (2006: 9), «ce sont souvent les Églises, issues des anciennes missions chrétiennes, qui ont pu devenir des espaces d'expression de la contestation politique, généralement parce qu'elles étaient présentes sur le territoire depuis suffisamment longtemps et qu'elles disposaient d'un réseau et d'une influence conséquente sur la population, 
voire sur le pouvoir en place». Voir à ce sujet Constantin et Coulon (1997) pour l'Afrique en général, et Hansen et Twaddle (1995) pour l'Afrique de l'Est.

82. On rappelle ici la spécificité du processus de démocratisation en Tanzanie, lancée par le parti unique et Nyerere en premier chef (commission Nyalali). Voir ce qu'en disent Lafargue \& Constantin (1996:147-148) : « Le débat sur le multipartisme s'avérait être l'apanage d'une élite politique et intellectuelle qui y voyait le moyen de se restructurer ou de se remobiliser, ou plus cyniquement de réinvestir des lieux de pouvoir, c'est-à-dire d'accumulation privée ».

83. Mazrui (1973)

84. Henry (1994), Mmuya \& Chaligha (1992), Mhina (1999), Constantin \& Lafargue (1996).

85. Baregu (1994).

86. Nipashe, 05/10/2005.

87. "Personne ne peut prétendre plus respecter Nyerere que le Parti de la Révolution" (Mtanzania, 21/10/2005).

88. Voir le commentaire du journaliste tanzanien Kidanka (2006), très engagé, qui aborde la question de la liberté de la presse sous l'angle du contrôle opéré par la présidence actuelle : "The President of Tanzania, Mr. Jakaya Kikwete is a born-propagandist. He has managed to lead the chorus of litany of praise to him, sung by the journalists. Little is said of Kikwete's blunders, and whenever a reporter cannot avoid to say it, there is a scapegoatPresidential Aides » (Le président de Tanzanie, M. Jakaya Kikwete, est un propagandiste né. Il a réussi à conduire le choeur de litanies de louanges qui lui sont adressées, chantées par les journalistes. On entend peu de choses sur les faux-pas de Kikwete, et chaque fois qu'il est difficile pour un journaliste de ne pas en parler, il y a un bouc émissaire - les aides du président).

89. Selon le père Andrzej G. Madry du Diocèse de Musoma, du bureau des relations avec l'extérieur, "the decision of the bishop of Musoma [to initiate Nyerere's cause] arose from the requests of many people who admired the devout Christian life of the Father of the Nation. They asked the bishop to start investigations that could lead to his beatification" (La décision de l'évêque de Musoma [d'initier la cause de Nyerere] vint des requêtes de beaucoup de gens qui admiraient la vie pieuse de chrétien du père de la nation. Ils ont demandé à l'évêque de commencer des investigations qui pourraient mener à sa béatification).

90. Constantin et Coulon (1997: 15).

91. Forster (1987), Heilman \& Kaiser (2002), Kaiser (1996), Ludwig (1996), Kihl (2006), Okema (1996).

92. Sur la question des mémoires anonymes de Nyerere et de la période socialiste, et de la tenue à l'écart des médias des voix critiques, je renvoie à un prochain article de l'auteure pour les Cahiers d'Études Africaines.

93. Entretien avec l'auteure, juin 2006.

94. Mes opinions sur Nyerere ne sont pas imprimables, et pour cela même, je préfèrerais les garder pour moi. Par exemple, si je vous disais que cet homme est responsable de la plupart des malheurs qui touchent les Tanzaniens aujourd'hui, vous ne me croiriez probablement pas. Comme la corruption institutionnalisée, l'arrogance sans limite du parti au pouvoir et de ses leaders, la pauvreté écrasante, etc. ». 
95. The Guardian, 05/03/2007. Voir aussi l'article paru dans The Guardian, 24/01/2006 : "I ask myself, why is there lack of enthusiasm among Catholics concerning Nyerere's process to sainthood? You can hardly hear ordinary people, expressing their satisfaction concerning Nyerere's process to sainthood. This is not because they hated him or he was not a good person, but simply because they fail to associate his spiritual life and the process to sanctity" (Je me demande: pourquoi y a-t-il un manque d'enthousiasme concernant le procès en sainteté de Nyerere? On entend rarement les gens ordinaires exprimer leur satisfaction concernant le procès en sainteté de Nyerere. Ce n'est pas parce ce qu'ils le détestaient, ou qu'il n'était pas une bonne personne, mais simplement parce qu'ils n'arrivent pas à associer sa vie spirituelle et le procès en sainteté).

96. Centlivres (2001).

INDEX

Index géographique : Tanzania | Tanzanie

\section{AUTEUR}

\section{MARIE-AUDE FOUÉRÉ}

Docteur en anthropologie sociale de l'École des Hautes en Sciences Sociales (EHESS) à Paris et attachée temporaire d'enseignement et de recherches (ATER). 